\title{
THE RADIO/GAMMA-RAY CONNECTION IN ACTIVE GALACTIC NUCLEI IN THE ERA OF THE FERMI LARGE AREA TELESCOPE
}

M. Ackermann ${ }^{1}$, M. Ajello ${ }^{1}$, A. Allafort ${ }^{1}$, E. Angelakis ${ }^{2}$, M. Axelsson ${ }^{3,4,5}$, L. Baldini ${ }^{6}$, J. Ballet $^{7}$, G. Barbiellini ${ }^{8,9}$, D. Bastieri $^{10,11}$, R. Bellazzini ${ }^{6}$, B. Berenji ${ }^{1}$, R. D. Blandford ${ }^{1}$, E. D. Bloom ${ }^{1}$, E. Bonamente ${ }^{12,13}$, A. W. Borgland ${ }^{1}$, A. Bouvier ${ }^{14}$, J. Bregeon ${ }^{6}$, A. Brez ${ }^{6}$, M. Brigida ${ }^{15,16}$, P. Bruel ${ }^{17}$, R. Buehler ${ }^{1}$, S. Buson ${ }^{10,11}$, G. A. Caliandro ${ }^{18}$, R. A. Cameron ${ }^{1}$, A. Cannon ${ }^{19,20}$, P. A. Caraveo ${ }^{21}$, J. M. Casandjian ${ }^{7}$, E. Cavazzuti ${ }^{22}$, C. Cecchi $^{12,13}$, E. Charles $^{1}$, A. Chekhtman ${ }^{23,57}$, C. C. Cheung ${ }^{24,57}$, S. Ciprini ${ }^{13}$, R. Claus ${ }^{1}$, J. Cohen-Tanugi ${ }^{25}$, S. Cutini ${ }^{22}$, F. De Palma ${ }^{15,16}$, C. D. Dermer ${ }^{26}$, E. do Couto e Silva ${ }^{1}$, P. S. Drell ${ }^{1}$, R. Dubois ${ }^{1}$, D. Dumora ${ }^{27}$, L. Escande ${ }^{27,28}$, C. Favuzzi $^{15}, 16$,

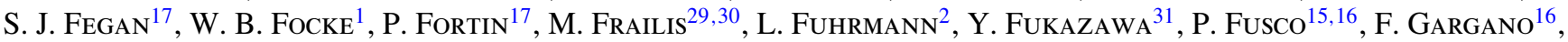
D. Gasparrini ${ }^{22}$, N. Gehrels ${ }^{19}$, N. Giglietto ${ }^{15}, 16$, P. Giommi ${ }^{22}$, F. Giordano ${ }^{15,16}$, M. Giroletti ${ }^{32,58}$, T. Glanzman ${ }^{1}$, G. Godfrey $^{1}$, P. Grandi ${ }^{33}$, I. A. Grenier ${ }^{7}$, S. Guiriec ${ }^{34}$, D. Hadasch ${ }^{18}$, M. Hayashida ${ }^{1}$, E. Hays ${ }^{19}$, S. E. Healey ${ }^{1}$, G. Jóhannesson ${ }^{35}$, A. S. Johnson ${ }^{1}$, T. KAMAe ${ }^{1}$, H. KATAgiri ${ }^{31}$, J. KATAOKA ${ }^{36}$, J. KNÖDlSEder ${ }^{37}$, M. Kuss ${ }^{6}$, J. LANDE ${ }^{1}$,

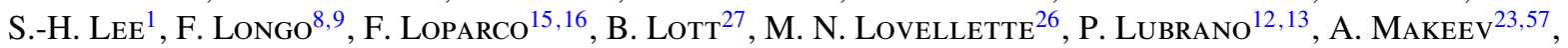
W. MaX-Moerbeck ${ }^{38}$, M. N. Mazziotta ${ }^{16}$, J. E. McEnery ${ }^{19,39}$, J. Mehault $^{25}$, P. F. Michelson ${ }^{1}$, T. Mizuno $^{31}$, C. Monte $^{15,16}$,

M. E. Monzani ${ }^{1}$, A. Morselli ${ }^{40}$, I. V. Moskalenko ${ }^{1}$, S. Murgia ${ }^{1}$, M. Naumann-Godo ${ }^{7}$, S. Nishino ${ }^{31}$, P. L. Nolan ${ }^{1}$, J. P. Norris ${ }^{41}$, E. Nuss ${ }^{25}$, T. Ohsugi ${ }^{42}$, A. Okumura ${ }^{43}$, N. Omodei ${ }^{1}$, E. Orlando ${ }^{1,44}$, J. F. Ormes ${ }^{41}$, M. Ozaki $^{43}$, D. Paneque ${ }^{1,45}$, V. Pavlidou ${ }^{38,58}$, V. Pelassa ${ }^{25}$, M. Pepe ${ }^{12,13}$, M. Pesce-Rollins ${ }^{6}$, M. Pierbattista ${ }^{7}$, F. Piron $^{25}$,

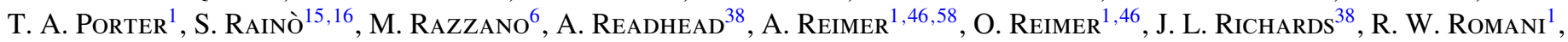
H. F.-W. Sadrozinski ${ }^{14}$, J. D. Scargle ${ }^{47}$, C. Sgrò ${ }^{6}$, E. J. Siskind ${ }^{48}$, P. D. Smith ${ }^{49}$, G. Spandre ${ }^{6}$, P. Spinelli ${ }^{15,16}$, M. S. Strickman ${ }^{26}$, D. J. Suson ${ }^{50}$, H. TAKahashi ${ }^{42}$, T. TANAKa ${ }^{1}$, G. B. TAYlor ${ }^{51}$, J. G. ThaYer ${ }^{1}$, J. B. ThaYeR $^{1}$, D. J. Thompson ${ }^{19}$, D. F. Torres ${ }^{18,52}$, G. Tosti ${ }^{12,13}$, A. Tramacere ${ }^{1,53,54}$, E. Troja $^{19,59}$, J. VAndenbroucke $^{1}$, G. Vianello $^{1,53}$,

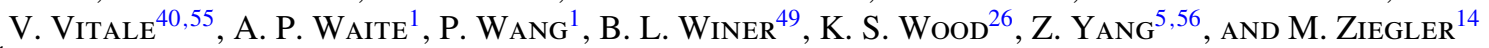

${ }^{1}$ W. W. Hansen Experimental Physics Laboratory, Kavli Institute for Particle Astrophysics and Cosmology, Department of Physics and SLAC National Accelerator Laboratory, Stanford University, Stanford, CA 94305, USA; afr@ slac.stanford.edu

${ }^{2}$ Max-Planck-Institut für Radioastronomie, Auf dem Hügel 69, 53121 Bonn, Germany

${ }^{3}$ Department of Astronomy, Stockholm University, SE-106 91 Stockholm, Sweden

${ }^{4}$ Lund Observatory, SE-221 00 Lund, Sweden

5 The Oskar Klein Centre for Cosmoparticle Physics, AlbaNova, SE-106 91 Stockholm, Sweden

${ }^{6}$ Istituto Nazionale di Fisica Nucleare, Sezione di Pisa, I-56127 Pisa, Italy

${ }^{7}$ Laboratoire AIM, CEA-IRFU/CNRS/Université Paris Diderot, Service d'Astrophysique, CEA Saclay, 91191 Gif sur Yvette, France

${ }^{8}$ Istituto Nazionale di Fisica Nucleare, Sezione di Trieste, I-34127 Trieste, Italy ${ }^{9}$ Dipartimento di Fisica, Università di Trieste, I-34127 Trieste, Italy

${ }^{10}$ Istituto Nazionale di Fisica Nucleare, Sezione di Padova, I-35131 Padova, Italy

${ }^{11}$ Dipartimento di Fisica "G. Galilei," Università di Padova, I-35131 Padova, Italy

12 Istituto Nazionale di Fisica Nucleare, Sezione di Perugia, I-06123 Perugia, Italy

${ }^{13}$ Dipartimento di Fisica, Università degli Studi di Perugia, I-06123 Perugia, Italy

${ }^{14}$ Santa Cruz Institute for Particle Physics, Department of Physics and Department of Astronomy and Astrophysics, University of California at Santa Cruz, Santa Cruz, CA 95064, USA

${ }^{15}$ Dipartimento di Fisica “M. Merlin" dell'Università e del Politecnico di Bari, I-70126 Bari, Italy

${ }^{16}$ Istituto Nazionale di Fisica Nucleare, Sezione di Bari, 70126 Bari, Italy

${ }^{17}$ Laboratoire Leprince-Ringuet, École polytechnique, CNRS/IN2P3, Palaiseau, France

${ }^{18}$ Institut de Ciencies de l'Espai (IEEC-CSIC), Campus UAB, 08193 Barcelona, Spain ${ }^{19}$ NASA Goddard Space Flight Center, Greenbelt, MD 20771, USA

${ }^{20}$ School of Physics, University College Dublin, Belfield, Dublin 4, Ireland

${ }^{21}$ INAF-Istituto di Astrofisica Spaziale e Fisica Cosmica, I-20133 Milano, Italy

${ }^{22}$ Agenzia Spaziale Italiana (ASI) Science Data Center, I-00044 Frascati (Roma), Italy

${ }^{23}$ College of Science, George Mason University, Fairfax, VA 22030, USA

${ }^{24}$ National Research Council Research Associate, National Academy of Sciences, Washington, DC 20001, USA

${ }^{25}$ Laboratoire de Physique Théorique et Astroparticules, Université Montpellier 2, CNRS/IN2P3, Montpellier, France

${ }^{26}$ Space Science Division, Naval Research Laboratory, Washington, DC 20375, USA

${ }^{27}$ Université Bordeaux 1, CNRS/IN2p3, Centre d'Études Nucléaires de Bordeaux Gradignan, 33175 Gradignan, France

${ }^{28}$ CNRS/IN2P3, Centre d'Études Nucléaires Bordeaux Gradignan, UMR 5797, 33175 Gradignan, France

${ }^{29}$ Dipartimento di Fisica, Università di Udine and Istituto Nazionale di Fisica Nucleare, Sezione di Trieste, Gruppo Collegato di Udine, I-33100 Udine, Italy

${ }^{30}$ Osservatorio Astronomico di Trieste, Istituto Nazionale di Astrofisica, I-34143 Trieste, Italy

${ }^{31}$ Department of Physical Sciences, Hiroshima University, Higashi-Hiroshima, Hiroshima 739-8526, Japan

${ }^{32}$ INAF Istituto di Radioastronomia, 40129 Bologna, Italy; giroletti@ira.inaf.it

33 INAF-IASF Bologna, 40129 Bologna, Italy

${ }^{34}$ Center for Space Plasma and Aeronomic Research (CSPAR), University of Alabama in Huntsville, Huntsville, AL 35899, USA

${ }^{35}$ Science Institute, University of Iceland, IS-107 Reykjavik, Iceland

${ }^{36}$ Research Institute for Science and Engineering, Waseda University, 3-4-1, Okubo, Shinjuku, Tokyo 169-8555 Japan

${ }^{37}$ Centre d'Étude Spatiale des Rayonnements, CNRS/UPS, BP 44346, F-30128 Toulouse Cedex 4, France

${ }^{38}$ Cahill Center for Astronomy and Astrophysics, California Institute of Technology, Pasadena, CA 91125, USA; pavlidou@ astro.caltech.edu

${ }^{39}$ Department of Physics and Department of Astronomy, University of Maryland, College Park, MD 20742, USA

${ }^{40}$ Istituto Nazionale di Fisica Nucleare, Sezione di Roma "Tor Vergata," I-00133 Roma, Italy

${ }^{41}$ Department of Physics and Astronomy, University of Denver, Denver, CO 80208, USA

${ }^{42}$ Hiroshima Astrophysical Science Center, Hiroshima University, Higashi-Hiroshima, Hiroshima 739-8526, Japan 


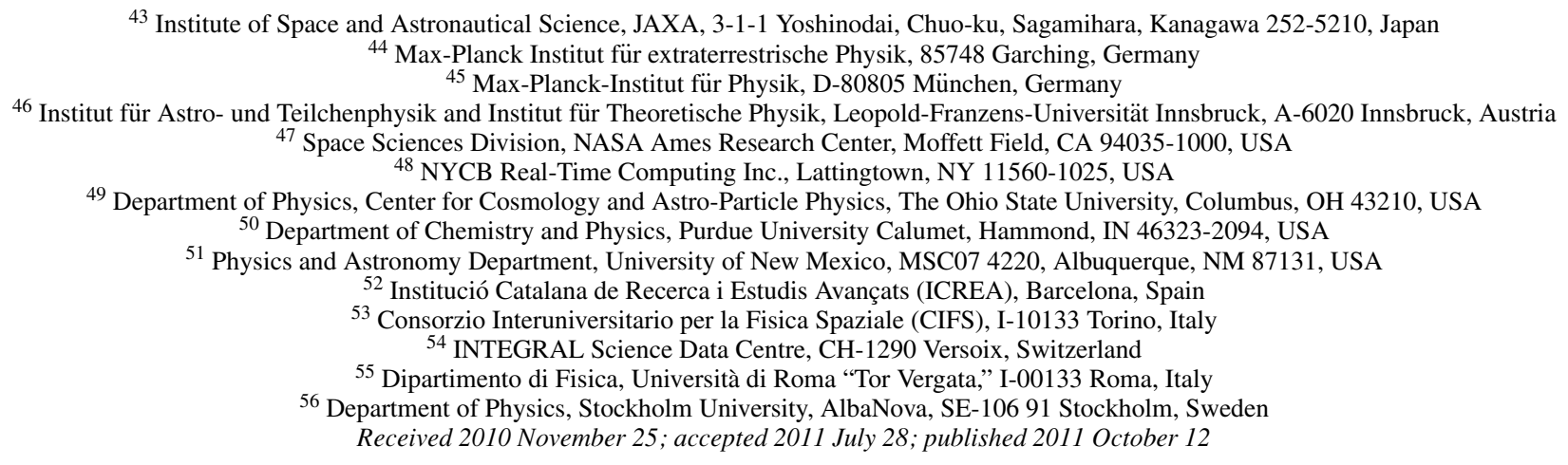

\begin{abstract}
We present a detailed statistical analysis of the correlation between radio and gamma-ray emission of the active galactic nuclei (AGNs) detected by Fermi during its first year of operation, with the largest data sets ever used for this purpose. We use both archival interferometric $8.4 \mathrm{GHz}$ data (from the Very Large Array and ATCA, for the full sample of 599 sources) and concurrent single-dish $15 \mathrm{GHz}$ measurements from the Owens Valley Radio Observatory (OVRO, for a sub sample of 199 objects). Our unprecedentedly large sample permits us to assess with high accuracy the statistical significance of the correlation, using a surrogate data method designed to simultaneously account for common-distance bias and the effect of a limited dynamical range in the observed quantities. We find that the statistical significance of a positive correlation between the centimeter radio and the broadband $(E>100 \mathrm{MeV})$ gamma-ray energy flux is very high for the whole AGN sample, with a probability of $<10^{-7}$ for the correlation appearing by chance. Using the OVRO data, we find that concurrent data improve the significance of the correlation from $1.6 \times 10^{-6}$ to $9.0 \times 10^{-8}$. Our large sample size allows us to study the dependence of correlation strength and significance on specific source types and gamma-ray energy band. We find that the correlation is very significant (chance probability $<10^{-7}$ ) for both flat spectrum radio quasars and BL Lac objects separately; a dependence of the correlation strength on the considered gamma-ray energy band is also present, but additional data will be necessary to constrain its significance.
\end{abstract}

Key words: BL Lacertae objects: general - galaxies: active - galaxies: jets - gamma rays: galaxies - radio continuum: galaxies - quasars: general

Online-only material: color figures, machine-readable table

\section{INTRODUCTION}

After more than one year of scanning the gamma-ray sky by the Large Area Telescope (LAT) on board the Fermi Gammaray Space Telescope (Fermi), the most extreme class of active galactic nuclei (AGNs), blazars (used to refer collectively to BL Lac objects, hereafter BL Lacs, and flat spectrum radio quasars, hereafter FSRQs), remains among the most numerous gamma-ray source populations. Indeed, the First Fermi-LAT catalog of gamma-ray sources (hereafter 1FGL; Abdo et al. 2010b) includes more than 1400 sources and about half of them are believed to be AGNs (Abdo et al. 2010c) with most of them identified via radio catalogs (e.g., CRATES; Healey et al. 2007). More than 370 high-latitude $\left(|b|>10^{\circ}\right)$ sources in the 1FGL remain unidentified.

Blazars have been observed to emit at all energies, from the radio band up to very high energy gamma rays. Many of the gamma-ray blazars detected so far appear to emit the bulk of their total radiative output at gamma-ray energies. Strong variability across the whole electromagnetic spectrum and on various timescales is considered as one of the most intriguing properties of this source type. In particular their high-energy emission can easily vary by more than an order of magnitude

\footnotetext{
${ }^{57}$ Resident at Naval Research Laboratory, Washington, DC 20375, USA.

${ }^{58}$ Corresponding authors.

${ }^{59}$ NASA Postdoctoral Program Fellow, USA.
}

from one observing epoch to the next (e.g., Mukherjee et al. 1997; Abdo et al. 2010d), and variability timescales at high energies are mostly much shorter (even down to just a few minutes in the TeV band, e.g., Aharonian et al. 2007) than in the long wavelength bands.

The high inferred bolometric luminosities, rapid variability, and apparent superluminal motions observed from a range of blazars provide compelling evidence that the non-thermal emission of blazars originates from a region which is propagating relativistically along a jet directed at a small angle with respect to our line of sight.

Because most identified gamma-ray AGNs are classified as radio-loud objects, a luminosity correlation between those two wavebands appears possible. If proved true, constraints on the physics and location of the jet emission from such AGNs may be deduced. Many attempts have been made in the past to investigate correlations between radio (cm)- and gamma-ray luminosities of AGN (e.g., Stecker et al. 1993; Padovani et al. 1993; Salamon \& Stecker 1994; Taylor et al. 2007). However, the relation has not been conclusively demonstrated when all relevant biases and selection effects are taken into account (see, e.g., Mücke et al. 1997).

For example, while luminosities represent the intrinsic source property, as opposed to fluxes, the use of luminosities always introduces a redshift bias in samples which cover a wide distance range since luminosities are strongly correlated with redshift (Elvis et al. 1978). Such redshift dependence can be removed 
by means of a partial correlation analysis (see, e.g., Dondi \& Ghisellini 1995). On the other hand, intrinsic correlations between the gamma-ray and radio luminosities may be smeared out, or even lost in the corresponding flux diagrams whereas artificial flux-flux correlations can be induced due to the effect of a common distance modulation of gamma-ray and radio luminosities (the "common-distance" bias; see, e.g., Pavlidou et al. 2011).

Samples that are strongly sensitivity limited restrict the populated region in the luminosity-luminosity diagram to a narrow band, thereby causing serious biases. Therefore, Feigelson \& Berg (1983) proposed to include all upper limits to avoid artificial correlations and incorrect conclusions (Schmitt 1985). However, upper limits are usually not distributed randomly in the flux-flux or luminosity-luminosity plane, but are localized in a particular area. In this case, a survival analysis may give misleading results (Isobe 1989). Furthermore, this analysis cannot account for biases caused by misidentification of sources or by truncation effects. Finally, the use of rank correlation tests (e.g., Kendall's $\tau$, Spearman rank correlation coefficient $\rho$ ) complicates the inclusion of observational uncertainties.

Another problem is the data and source selection. Blazars are inherently variable sources in the radio as well as the gammaray band on a broad range of timescales. Simultaneous observations are therefore the only appropriate data for a correlation analysis. However, due to the lack of such data, the mean (e.g., Padovani et al. 1993) or the brightest flux values (e.g., Dondi \& Ghisellini 1995) have often been used instead. As a consequence the dynamical range in the luminosity-luminosity plane is significantly reduced in those cases, and can hence mimic a correlation (Mücke et al. 1997).

The question of a correlation between the radio and $\mathrm{GeV}$ band on the basis of Fermi data has recently generated a lot of interest and has been the subject of a series of investigations (Kovalev et al. 2009; Ghirlanda et al. 2010, 2011; Mahony et al. 2010). However, these studies have been generally limited to a small fraction of the Fermi-detected AGNs and have used non-simultaneous or quasi-simultaneous measurements. Moreover, these works have primarily addressed the issue of the apparent strength of the correlation, rather than that of its intrinsic significance, which requires a dedicated method of statistical analysis. In this paper, we will use the term "apparent correlation strength" for measures of the tightness of a correlation between radio and gamma-ray fluxes (such as various correlation coefficients) as seen in the raw data, without applying any correction or significance assessment to address common-distance bias and the limits on the measured fluxes (the issue of "censored data"). In contrast, we will use the term "intrinsic correlation" for the physical correlation between radio and gamma-ray (time-averaged) luminosities, in the limit of an infinite survey, and "intrinsic correlation significance" for the statistical significance of the claim that a specific data set exhibits a non-zero intrinsic correlation.

In this paper, we revisit this topic exploiting for the first time the Fermi-LAT data in full, in two ways. First, we make use of archival data for about 600 sources, a data set more than twice as large as that used in Ghirlanda et al. (2010) and Mahony et al. (2010). Second, we take advantage of the large set of concurrent measurements provided by the Owens Valley Radio Observatory (OVRO) monitoring program (Richards et al. 2010). The pre-Fermi-launch OVRO sample included $\sim 200$ blazars that are included in the 1FGL catalog, and for which average $15 \mathrm{GHz}$ fluxes measured concurrently with the 1FGL gamma-ray fluxes can be calculated. In addition, we exploit a new statistical method (Pavlidou et al. 2011) to assess the significance of the correlation coefficients.

The paper is structured as follows: in Section 2, we present the gamma-ray and radio data and the association procedure; the results are presented in Section 3 and discussed in Section 4 using a dedicated statistical analysis based on the method of surrogate data. A more general discussion is given in Section 5 and the main conclusions are summarized in Section 6.

In the following, we use a $\Lambda$ CDM cosmology with $h=0.71$, $\Omega_{m}=0.27$, and $\Omega_{\Lambda}=0.73$ (Komatsu et al. 2009). The radio spectral index is defined such that $S(v) \propto v^{-\alpha}$ and the gammaray photon index $\Gamma$ such that $d N_{\text {photon }} / d E \propto E^{-\Gamma}$.

\section{OBSERVATIONS AND DATA SET}

\subsection{Gamma-Ray Data}

The gamma-ray sources in the present paper are a subset of those in the First Fermi-LAT catalog (1FGL; Abdo et al. 2010b). The 1FGL is a catalog of high-energy gamma-ray sources detected by the LAT during the first 11 months of the science phase of Fermi, i.e., between 2008 August 4 and 2009 July 4. The procedures used in producing the 1FGL catalog are discussed in detail in Abdo et al. (2010b); in total, the 1FGL contains 1451 sources detected and characterized in the $100 \mathrm{MeV}$ to $100 \mathrm{GeV}$ range and belonging to a number of populations of gamma-ray emitters.

In general, associations of gamma-ray sources with lowerenergy counterparts necessarily rely on a spatial coincidence between the two. A firm counterpart identification requires the search for correlated variability, which is a major effort in the case of AGNs; therefore, only five AGNs are listed as firm identifications by Abdo et al. (2010b), although ongoing studies will undoubtedly expand this set. For the rest, associations in 1FGL use a method for finding correspondence between LAT sources and AGNs based on the calculation of association probabilities using a Bayesian approach implemented in the gtsrcid tool included in the LAT ScienceTools package. A detailed description and a complete list of the source catalogs used by gtsrcid to draw candidate counterparts can be found in Abdo et al. (2010b).

The set of all high-latitude $\left(|b|>10^{\circ}\right)$ 1FGL sources with an AGN association from gtsrcid constitutes the First LAT AGN Catalog (1LAC; Abdo et al. 2010c). Some LAT sources are associated with multiple AGNs, and consequently, the catalog includes 709 AGN associations for 671 distinct 1FGL sources. Each source has an association probability $P$, evaluated by examining the local density of counterparts from a number of source catalogs in the vicinity of the LAT source. The main catalogs used are the Combined Radio All-sky Targeted Eight GHz Survey (CRATES; Healey et al. 2007), the Candidate Gamma-Ray Blazar Survey (CGRaBS; Healey et al. 2008), and the Roma-BZCAT (Massaro et al. 2009). Since a few gamma-ray sources have more than one possible association, and not all associations are highly significant, Abdo et al. (2010c) have further defined an AGN "clean" sample consisting of those AGNs that (1) are the sole AGNs associated with the corresponding 1FGL gamma-ray source and (2) have an association probability $P \geqslant 80 \%$; a few sources, "flagged" in the 1FGL catalog as exhibiting some problem, have also been discarded and do not belong in the 1LAC clean sample. This clean sample contains 599 AGNs. In the following analysis, 
whenever we mention the 1LAC sample, we will always be referring to the clean sample even if we do not state so explicitly.

For each source in the 1FGL (and hence in the 1LAC), Abdo et al. (2010b) have first obtained good estimates of the significance and the overall spectral slope $\Gamma$. Then, in order to obtain good estimates of the energy flux, each of the five energy bands (from 100 to $300 \mathrm{MeV}, 300 \mathrm{MeV}$ to $1 \mathrm{GeV}, 1$ to $3 \mathrm{GeV}$, 3 to $10 \mathrm{GeV}$, and 10 to $100 \mathrm{GeV}$ ) has been fit independently, fixing the spectral index of each source to $\Gamma$ as derived from the fit over the full interval; finally, the sum of the energy flux in the five bands provided a reliable estimate of the overall flux.

In sources with a poorly measured flux (88/599), Abdo et al. (2010b) replaced the value from the likelihood analysis with a $2 \sigma$ upper limit. However, since these sources are significantly detected when the full band is considered, we estimated their energy fluxes from the flux densities at the pivot energies given by Abdo et al. (2010b) and using the tabulated photon indices and the relative uncertainties on the corresponding quantities. All the obtained data are consistent with the $2 \sigma$ limits and so have been used for our analysis.

We maintain the 1LAC classification of each AGN on the basis of its optical spectrum either as an FSRQ or a BL Lac using the same scheme as in CGRaBS (Healey et al. 2008). In particular, following Stocke et al. (1991), Urry \& Padovani (1995), and Marchã et al. (1996), an object is classified as a BL Lac if the equivalent width (EW) of the strongest optical emission line is $<5 \AA$, the optical spectrum shows a Ca II $\mathrm{H} / \mathrm{K}$ break ratio $C<0.4$, and the wavelength coverage of the spectrum satisfies $\left(\lambda_{\max }-\lambda_{\min }\right) / \lambda_{\max }>1.7$ in order to ensure that at least one strong emission line would have been detected if it were present.

In addition to the optical spectrum classification, the 1LAC blazars are also classified based on the position of the synchrotron peak, following the scheme proposed by Abdo et al. (2010a); we therefore also consider the three following spectral types: low-synchrotron-peaked (LSP, $v_{\text {peak }}^{\mathrm{S}}<10^{14} \mathrm{~Hz}$ ), intermediate-synchrotron-peaked (ISP, $10^{14} \mathrm{~Hz}<v_{\text {peak }}^{\text {S }}<$ $10^{15} \mathrm{~Hz}$ ), or high-synchrotron-peaked (HSP, $v_{\text {peak }}^{\mathrm{S}}>10^{15} \mathrm{~Hz}$ ) blazars. Although the two classification schemes do have some degeneracy (e.g., HSP sources are largely BL Lacs, while most FSRQs are LSP sources), it is relevant to discuss them both, as the spectral classification is linked to the physical process (synchrotron radiation) responsible for the low-frequency emission.

In our study we will of course be using only sources that have been associated with a low-energy AGN counterpart. However, we note that 1FGL also contains 374 unassociated sources. If some of these sources are AGNs that were not associated with a lower-energy counterpart because they happen to be too faint in radio, then this could potentially introduce a bias in our assessment of the radio/gamma flux correlations. In Figure 1, we show normalized histograms of the gamma-ray fluxes of the high-latitude $\left(|b|>10^{\circ}\right)$ AGNs and of the high-latitude unassociated sources. Although in both distributions the sources tend to cluster in the low flux bins, this effect is much more pronounced in the unassociated gamma-ray sources, and there is strong statistical evidence that the two samples are not drawn from the same population (K-S probability of $4.3 \times 10^{-13}$ ). This makes it unlikely that we significantly overestimate the strength of the correlation because of the existence of yet-unassociated, radio-faint and gamma-ray bright blazars.

On the other hand, in any given radio flux limited sample there are sources that are radio bright and gamma-ray quiet (see, e.g.,

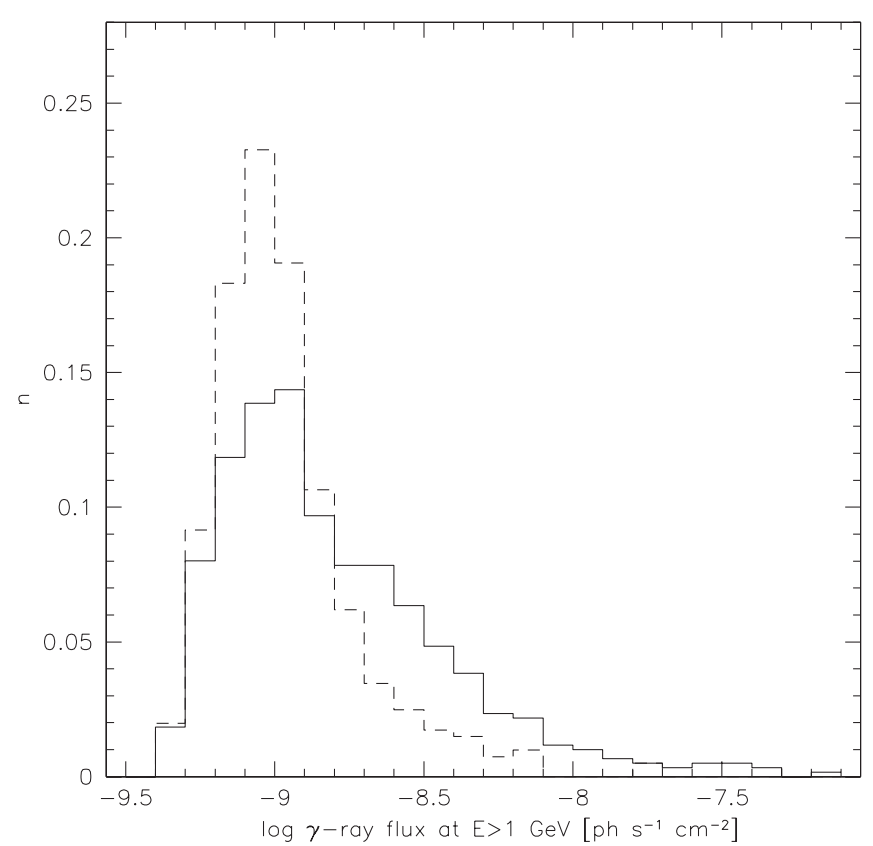

Figure 1. Normalized distribution of the gamma-ray photon flux for highlatitude $\left(|b| \geqslant 10^{\circ}\right)$ associated (solid line) and unassociated (dashed line) 1FGL sources.

Section 2.2.2 below for the case of the OVRO sample). This fact can be the consequence of long-term variability and/or low duty cycle in gamma rays (Ghirlanda et al. 2011); in any case, in this paper we only deal with the sources detected by LAT.

\subsection{Radio Data}

In Table 1, we list the radio flux densities used for the present work, along with some basic information on the sources (position, optical and spectral type, redshift). In particular, we give the archival $8 \mathrm{GHz}$ interferometric flux density in Column 8 (with the corresponding reference in Column 9) and the $15 \mathrm{GHz}$ single-dish flux density, when available, in Columns 10-12. A summary of the details of the relevant observations are given in the following subsections.

\subsubsection{CRATES/Other Catalogs}

For all sources in the 1LAC, we were able to collect interferometric measurements of the historic radio flux density. This provides us with the largest database of radio and gammaray measurements ever obtained and we use it for a discussion of the correlation between the two bands.

Most of these data come from CRATES (478 sources) or CRATES-like (96 sources) observations. The CRATES catalog (Healey et al. 2007) contains precise positions, 8.4 GHz flux densities, and radio spectral indices for over 11,000 flatspectrum sources over the entire $|b|>10^{\circ}$ sky. In the region $\delta>-40^{\circ}$, the $8.4 \mathrm{GHz}$ data were obtained with the Very Large Array (VLA) in its largest (A) configuration, and the spectral indices were determined by comparing the $8.4 \mathrm{GHz}$ flux density and the $1.4 \mathrm{GHz}$ flux density from the NRAO VLA Sky Survey (NVSS; Condon et al. 1998). In the region $\delta<-40^{\circ}$, the $8.4 \mathrm{GHz}$ data were obtained with ATCA in a variety of large configurations $(6 \mathrm{~A} / \mathrm{C} / \mathrm{D}, 1.5 \mathrm{~B} / \mathrm{C} / \mathrm{D})$, and the spectral indices were determined by comparing the $8.4 \mathrm{GHz}$ flux density and the 
Table 1

Source List and Radio Data

\begin{tabular}{|c|c|c|c|c|c|c|c|c|c|c|c|}
\hline 1FGL Name & Other Name & $\begin{array}{c}\text { R.A. } \\
\left({ }^{\circ}\right)\end{array}$ & $\begin{array}{c}\text { Decl. } \\
\left({ }^{\circ}\right)\end{array}$ & Opt. Type & SED Type & $z$ & $\begin{array}{c}S_{8} \\
(\mathrm{mJy})\end{array}$ & Ref. & $\begin{array}{c}S_{15, \text { lyear mean }} \\
(\mathrm{mJy})\end{array}$ & $\begin{array}{c}S_{15, \text { lyear peak }} \\
(\mathrm{mJy})\end{array}$ & $\begin{array}{l}S_{15, \text { non-c. }} \\
(\mathrm{mJy})\end{array}$ \\
\hline 1FGL J0000.9-0745 & CRATES J0001-0746 & 0.32512 & -7.77417 & BLL & LSP & $\ldots$ & 116.2 & $\mathrm{Cr}$ & & & \\
\hline 1FGL J0004.7-4737 & PKS 0002-478 & 1.14867 & -47.60517 & FSRQ & LSP & 0.88 & 780.4 & $\mathrm{Cr}$ & & $\ldots$ & \\
\hline 1FGL J0005.7+3815 & B2 $0003+38 \mathrm{~A}$ & 1.48825 & 38.33755 & FSRQ & LSP & 0.229 & 1078 & $\mathrm{Cr}$ & 541.9 & 703.3 & 541.2 \\
\hline 1FGL J0008.9+0635 & CRATES J0009+0628 & 2.26638 & 6.47256 & BLL & LSP & $\ldots$ & 196.5 & $\mathrm{Cr}$ & $\ldots$ & $\ldots$ & $\ldots$ \\
\hline 1FGL J0011.1+0050 & CGRaBS J0011+0057 & 2.87667 & 0.96439 & FSRQ & LSP & 1.492 & 278.7 & $\mathrm{Cr}$ & 249.3 & 364.8 & 205.6 \\
\hline 1FGL J0013.1-3952 & PKS 0010-401 & 3.24962 & -39.90717 & BLL & $\ldots$ & $\ldots$ & 1554 & $\mathrm{Cr}$ & $\ldots$ & $\ldots$ & $\ldots$ \\
\hline 1FGL J0013.7-5022 & BZB J0014-5022 & 3.54675 & -50.37575 & BLL & HSP & & 13.4 & $\mathrm{~N}$ & $\ldots$ & $\ldots$ & $\ldots$ \\
\hline 1FGL J0017.4-0510 & CGRaBS J0017-0512 & 4.39925 & -5.21158 & FSRQ & LSP & 0.227 & 225.2 & $\mathrm{Cr}$ & 297.8 & 497.3 & 173.1 \\
\hline 1FGL J0018.6+2945 & BZB J0018+2947 & 4.61562 & 29.79178 & BLL & HSP & $\ldots$ & 3.5 & $\mathrm{~F}$ & $\ldots$ & $\ldots$ & $\ldots$ \\
\hline 1FGL J0019.3+2017 & PKS 0017+200 & 4.90771 & 20.36267 & BLL & LSP & $\ldots$ & 1233 & $\mathrm{Cr}$ & 649.9 & 753.8 & 537.4 \\
\hline
\end{tabular}

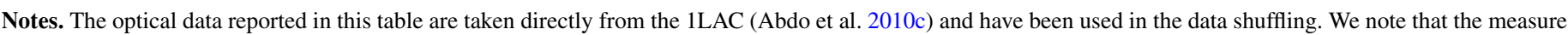

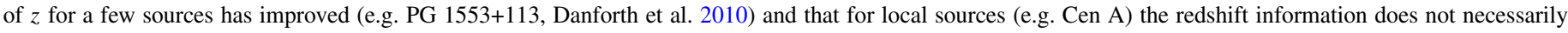
provide the most accurate estimate of the actual distance.

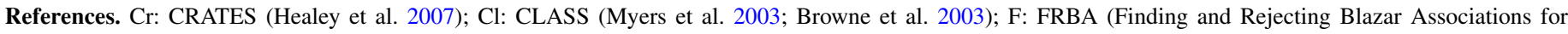

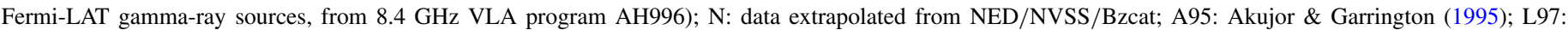

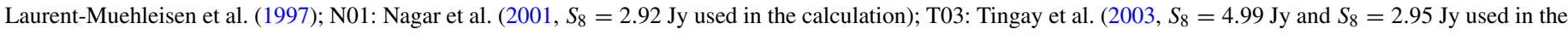
calculation for 1FGL J0522.8-3632 and 1FGL J1325.6-4300, respectively); U84: Unger et al. (1984).

(This table is available in its entirety in a machine-readable form in the online journal. A portion is shown here for guidance regarding its form and content.)

$843 \mathrm{MHz}$ flux density from the Sydney University Molonglo Sky Survey (SUMSS; Mauch et al. 2003) ${ }^{60}$

The data for sources that are not in CRATES are often of identical or very similar quality to those for CRATES sources. For example, $8.4 \mathrm{GHz}$ data from the Cosmic Lens All-Sky Survey (CLASS; Myers et al. 2003; Browne et al. 2003), from which the CRATES catalog obtained much of its northern hemisphere data in the first place, were all taken with the VLA in the A configuration. Similarly, the PMN-CA catalog ${ }^{61}$ of over 6600 radio sources was compiled from $8.6 \mathrm{GHz}$ data obtained with ATCA in the $6 \mathrm{~A}, 6 \mathrm{C}$, and $6 \mathrm{D}$ configurations. As a result, the radio flux densities and spectral indices of most non-CRATES sources can still be compared directly to those of true CRATES sources without introducing any systematic errors or biases.

For 19 sources, for which $8.4 \mathrm{GHz}$ VLA or ATCA measurements are not available, we extrapolate from lower frequency interferometric measurements (e.g., those reported from the Roma-BZCAT; Massaro et al. 2009). The spectral indices used for the extrapolation are those available from NED; when none was available, it was conventionally set to $\alpha=0.0$.

Finally, there are six sources in the 1LAC that possess a significant amount of extended radio emission (such as the misaligned AGNs discussed by Abdo et al. 2010e) and escape the selection criteria of CRATES and similar surveys. However, these are all rather well-known radio sources, and it has been straightforward to obtain interferometric measurements of their radio core flux density from the literature, either directly or with trivial calculations (e.g., interpolation).

\subsubsection{OVRO}

Since late 2007, the OVRO $40 \mathrm{~m}$ Telescope has been engaged in a blazar monitoring program to support the Fermi-LAT (Richards et al. 2010). In this program, all 1158 CGRaBS blazars north of declination $-20^{\circ}$ have been observed approximately

\footnotetext{
60 Strictly speaking, the ATCA observations were performed at $8.6 \mathrm{GHz}$, and the flux densities were converted to $8.4 \mathrm{GHz}$ by interpolation using the spectral index, but even for a very inverted source $(\alpha=-1)$, this represents an adjustment of $<3 \%$ to the flux density.

61 Survey results can be downloaded from

http://www.parkes.atnf.csiro.au/observing/databases/pmn/casouth.pdf
}

twice per week or more frequently since 2007 June (Healey et al. 2008). Gamma-ray blazars and other sources detected by Fermi have been added to the program which makes the total number of monitored sources close to 1500 . Of these sources, 199 appear as "clean" associations in the 1LAC catalog.

The OVRO flux densities are measured in a single $3 \mathrm{GHz}$ wide band centered on $15 \mathrm{GHz}$. Observations were performed using azimuth double switching as described in Readhead et al. (1989), which removes much atmospheric and ground interference. The relative uncertainties in flux density result from a $5 \mathrm{mJy}$ typical thermal uncertainty in quadrature with a $1.6 \%$ systematic uncertainty. The absolute flux density scale is calibrated to about $5 \%$ via observations of the steady calibrator 3C 286, using the Baars et al. model (Baars et al. 1977). A complete description of the OVRO program, population studies of the radio variability, their relation with other physical properties, and a study of the time relation between radio and gamma-ray emission are presented in a series of dedicated publications (Richards et al. 2010; Pavlidou et al. 2011; W. Max-Moerbeck et al. 2011, in preparation).

Because the Fermi-LAT flux densities used in this study represent time averages over the observation period, we produce estimates of the $15 \mathrm{GHz}$ time average flux density from the OVRO data for each source by linearly interpolating between successive light curve values, integrating between the start and end dates, then dividing by the time interval. For the 11 month data here, the start date was midnight 2008 August 4 (MJD 54682) and the end date was midnight 2009 July 4 (MJD 55016). Hereafter, we will be referring to average $15 \mathrm{GHz}$ radio fluxes obtained in this manner as the OVRO concurrent data.

The normalized distribution of average fluxes of the OVRO subset is shown in Figure 2, overplotted with the distribution of average fluxes, obtained in the same manner, of gamma-ray quiet CGRaBS sources north of declination $-20^{\circ}$. The sources which are also in 1LAC have generally higher $15 \mathrm{GHz}$ average fluxes. However, there is substantial overlap between the two distributions, so the existence of sources with large fluxes at $15 \mathrm{GHz}$ but which are faint in gamma rays is not unexpected. Therefore, our expectation from the distribution of fluxes alone is that if a statistically significant correlation between radio and gamma-ray fluxes indeed exists, it will likely have a substantial scatter. 


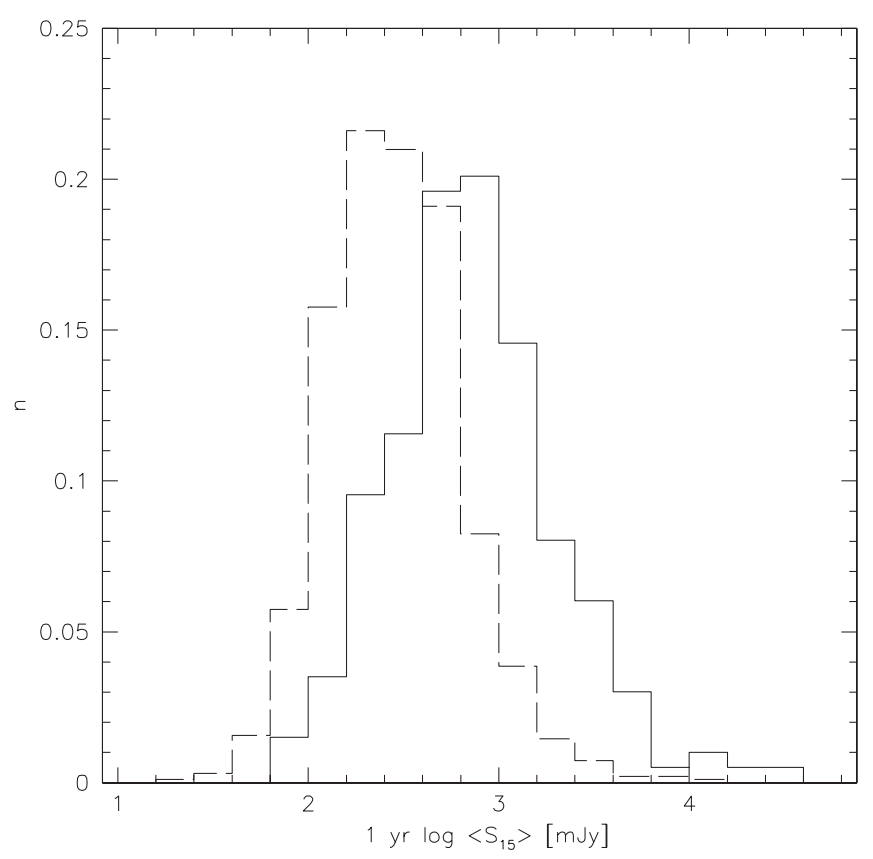

Figure 2. Normalized distributions of the average $15 \mathrm{GHz}$ flux densities for CGRABS sources north of declination $-20^{\circ}$, shown separately for gamma-ray associated (solid) and unassociated (dashed) sources.

\section{RESULTS}

In this section, we present the results of our search for possible correlations between radio flux densities and the gamma-ray photon flux for the sources in the 1LAC sample. In particular, in Section 3.1 we consider the full 1LAC sample and search for correlations with archival radio data, while in Section 3.2 we focus on the subset of sources observed at OVRO, considering both concurrent and archival radio data; finally, in Section 3.3 we present results for a subset of the 1LAC composed of sources detected in at least four individual energy bands. There are 599 sources in the 1LAC clean sample and 199 in the 1LAC-OVRO sample. The OVRO $15 \mathrm{GHz}$ concurrent fluxes are averaged (time integrated, as in the gamma-ray data) over the same interval as the LAT observations, and for all of sources considered here there exists gamma-ray variability on timescales shorter than the averaging period.

For each sample, we have compared the radio flux density to the 1 year gamma-ray energy flux at $E>100 \mathrm{MeV}$. Moreover, since we have unprecedentedly large data sets, we can also explore whether the strengths of any observed correlations are dependent on the gamma-ray energy band in which the flux is calculated or on the source spectral type. For this reason, we also compare radio flux densities to gamma-ray photon fluxes calculated in the single energy bands $100 \mathrm{MeV}<E<$ $300 \mathrm{MeV}, 300 \mathrm{MeV}<E<1 \mathrm{GeV}, 1 \mathrm{GeV}<E<3 \mathrm{GeV}$, $3 \mathrm{GeV}<E<10 \mathrm{GeV}$, and $10 \mathrm{GeV}<E<100 \mathrm{GeV}$. In each energy band, we consider only the sources that are significant in that band. Not every source is detected in all energy bands; actually, only a small minority is, i.e., 51/599 (8.5\%). As a consequence of their different spectral properties, FSRQs are generally more abundant in the lowest energy bands, while BL Lacs are more numerous in the most energetic ones. For instance, for the 1LAC sources, we have 128 FSRQs and 47 BL Lacs in the 100-300 MeV band, and 22 FSRQs and 99 BL Lacs in the 10-100 GeV band.

Since FSRQs and BL Lacs have different spectral properties and showed different behaviors in the preliminary analysis
(Abdo et al. 2009; Giroletti et al. 2010), we also tested the two populations separately, in addition to the full set of sources. Moreover, a classification based on the broadband spectral properties is physically more meaningful, so we also consider the populations of LSP, ISP, and HSP blazars.

In total, we have 36 combinations of source type and gammaray energy band for the 1LAC. For the OVRO sample, we have also the possibility to consider the radio data obtained at $15 \mathrm{GHz}$ during the same interval of the gamma-ray observations, both as mean and peak flux density measurements, and in a different time domain. For each combination, we produced a scatter plot of the radio versus gamma-ray flux densities and determined the Spearman's rank correlation $\rho$, which are presented in the following subsections.

The value of $\rho$ is characteristic of the strength of the correlation, and it can be related to the significance of an apparent correlation between radio and gamma-ray fluxes. However, an assessment of the statistical significance of an intrinsic correlation in each case (after the effects of a common distance and a limited dynamical range are accounted for) is nontrivial and cannot be based on a conventional assumption of unbiased samples. Therefore, we use simulations based on the method of surrogated data to evaluate the significance of intrinsic correlations and discuss them in Section 4.

\subsection{Full Sample}

The sources associated with the 1LAC members span over four orders of magnitude in radio flux density, ranging between a few mJy for the faintest BL Lacs to several 10's of Jy for the brightest quasars (e.g., 3C 273 and 3C 279). The flux density distributions for the whole population and divided by the source type are shown in Abdo et al. (2010c). The overall distribution shows a broad peak at $S \sim 800 \mathrm{mJy}$, which is the result of the combination of the two peaks of the single population distributions, with BL Lacs peaking around $S \sim 400 \mathrm{mJy}$ and FSRQs at $S \sim 1300 \mathrm{mJy}$. In gamma rays, the energy fluxes span over three orders of magnitude (between $4.8 \times 10^{-12}$ and $6.6 \times 10^{-10} \mathrm{erg} \mathrm{cm}^{-2} \mathrm{~s}^{-1}$ at $E>100 \mathrm{MeV}$ ), with BL Lacs typically fainter than FSRQs; the mean photon fluxes at $E>100 \mathrm{MeV}$ are $8.5 \times 10^{-8}$ photons $\mathrm{cm}^{-2} \mathrm{~s}^{-1}$ and $2.9 \times 10^{-8}$ photons $\mathrm{cm}^{-2} \mathrm{~s}^{-1}$ for FSRQs and BL Lacs, respectively (Abdo et al. 2010c).

We show the gamma-ray and radio flux scatter plots for the 1LAC sources in Figures 3-5. Each figure shows a collection of panels showing various combinations of the 1FGL gamma-ray flux and radio historical flux density. In particular, Figure 3 shows the gamma-ray energy flux versus radio flux density for all sources (top left panel), sources divided by the optical type (FSRQ and BL Lacs in the center and right top panels, respectively), and sources divided by the spectral type (bottom row, with LSP, ISP, and HSP in the left, middle, and right panels, respectively); in Figures 4 and 5, we show the gamma-ray photon flux versus radio flux in the five individual LAT energy bands (left to right), divided by the source type: in Figure 4, the top row shows all sources, the middle one shows FSRQs, and the bottom one BL Lacs; in Figure 5, top, middle, and bottom rows are for the three different synchrotron peak classes: LSP, ISP, and HSP blazars, respectively. Symbols in gray show sources for which a redshift is not available.

We report the correlation coefficients between radio and gamma-ray flux for the full sample in Table 2, divided by the source type and energy band, and we visualize them in Figure 6. In this figure, the correlation coefficients are shown 


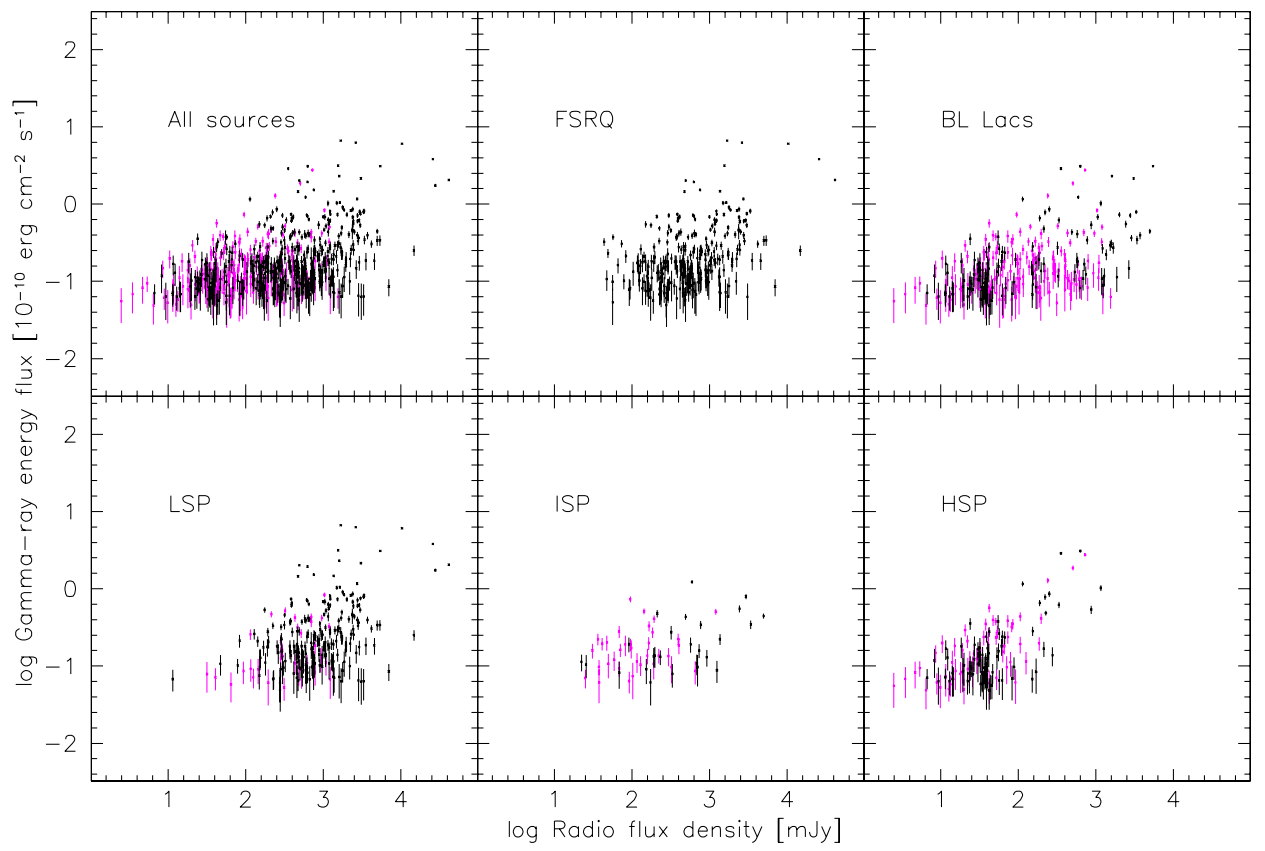

Figure 3. Broadband gamma-ray energy flux vs. $8 \mathrm{GHz}$ archival radio flux density for the 1LAC sample, divided by the source type. Top, from right to left: all AGNs, FSRQ, BL Lacs; bottom, from right to left: LSP, ISP, and HSP blazars. Sources with unknown redshift are shown in gray.

(A color version of this figure is available in the online journal.)

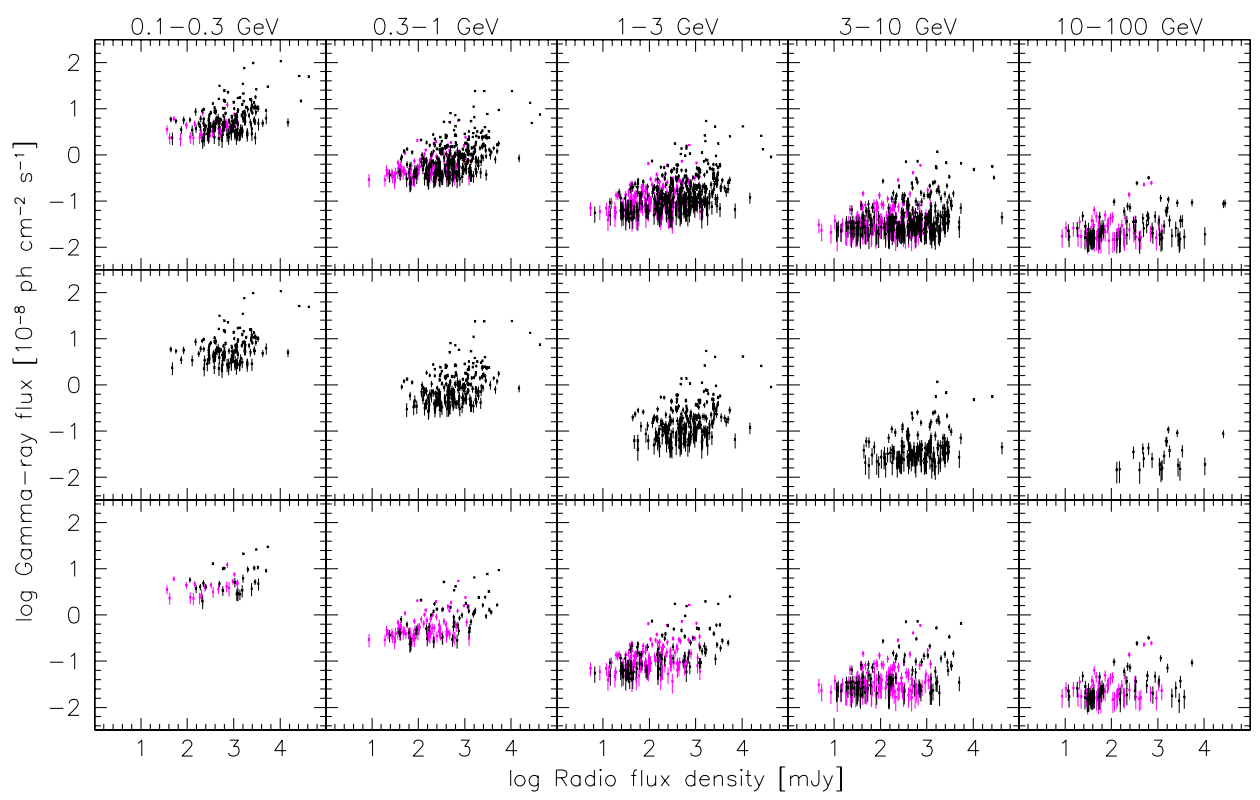

Figure 4. Gamma-ray photon flux vs. $8 \mathrm{GHz}$ archival radio flux density for the 1LAC sample, divided by the source optical type (top: all sources, middle: FSRQ, bottom: BL Lacs) and in energy bands. Sources with unknown redshift are shown in gray.

(A color version of this figure is available in the online journal.)

Table 2

Spearman Rank Correlation Coefficient $\rho$ for the 1LAC Clean Sample, Divided by the Source Type

\begin{tabular}{|c|c|c|c|c|c|c|c|c|c|c|c|c|}
\hline \multirow[t]{2}{*}{ Source Class } & \multicolumn{2}{|c|}{$E>0.1$} & \multicolumn{2}{|c|}{$0.1<E<0.3$} & \multicolumn{2}{|c|}{$0.3<E<1$} & \multicolumn{2}{|c|}{$1<E<3$} & \multicolumn{2}{|c|}{$3<E<10$} & \multicolumn{2}{|c|}{$10<E<100$} \\
\hline & $n$ & $\rho$ & $n$ & $\rho$ & $n$ & $\rho$ & $n$ & $\rho$ & $n$ & $\rho$ & $n$ & $\rho$ \\
\hline All sources & 599 & 0.43 & 193 & 0.41 & 384 & 0.51 & 493 & 0.43 & 373 & 0.31 & 127 & 0.30 \\
\hline FSRQ & 248 & 0.39 & 128 & 0.32 & 214 & 0.41 & 220 & 0.35 & 140 & 0.29 & 22 & 0.42 \\
\hline BL Lacs & 275 & 0.46 & 47 & 0.48 & 135 & 0.54 & 214 & 0.54 & 197 & 0.35 & 99 & 0.31 \\
\hline LSP & 242 & 0.40 & 133 & 0.37 & 200 & 0.42 & 219 & 0.38 & 158 & 0.39 & 35 & 0.35 \\
\hline ISP & 60 & 0.33 & 18 & 0.58 & 41 & 0.40 & 53 & 0.44 & 42 & 0.29 & 15 & 0.30 \\
\hline HSP & 129 & 0.55 & 12 & 0.16 & 47 & 0.62 & 87 & 0.64 & 88 & 0.57 & 64 & 0.55 \\
\hline
\end{tabular}

Note. For each energy band ( $E$ measured in $\mathrm{GeV}$ ), the number of significant sources $n$ and the correlation coefficient $\rho$ are given. 


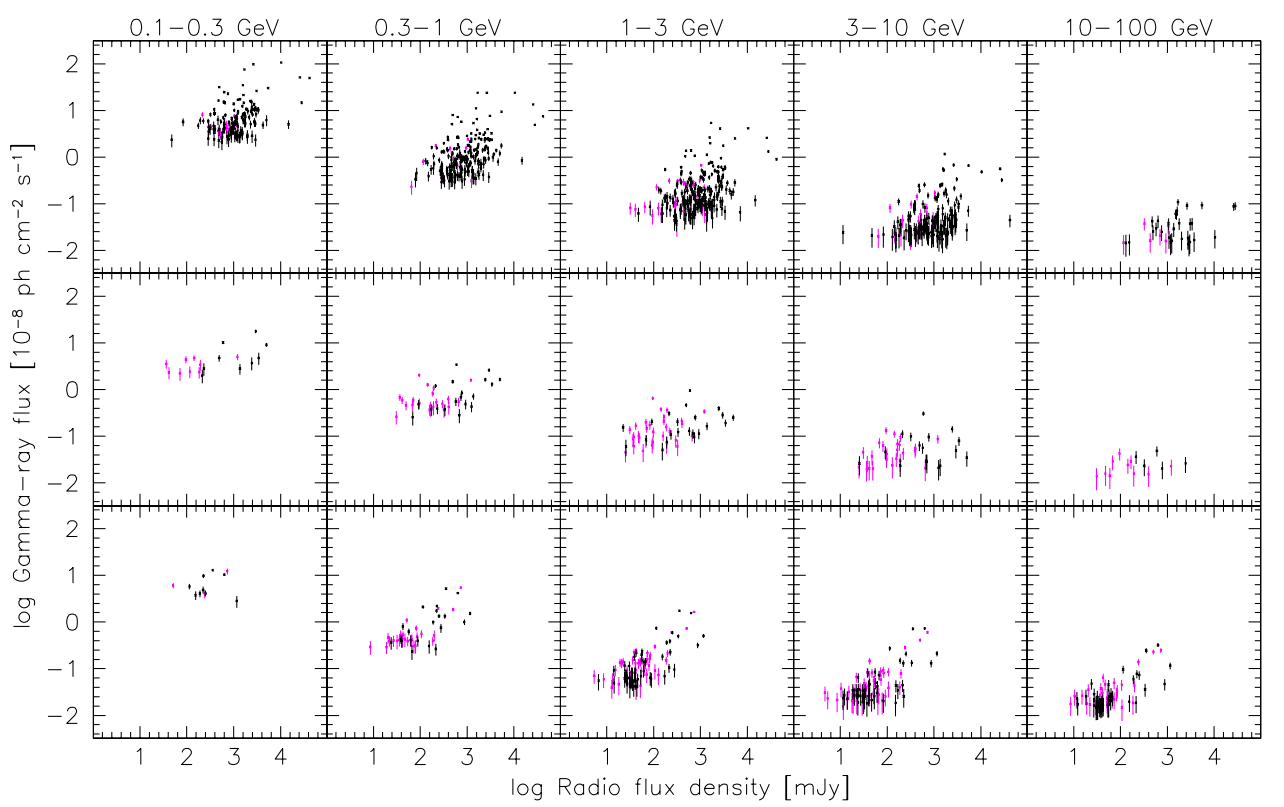

Figure 5. Gamma-ray photon flux vs. $8 \mathrm{GHz}$ archival radio flux density for the 1LAC sample, divided by the source spectral type (top: LSP, middle: ISP, bottom: HSP) and in energy bands. Sources with unknown redshift are shown in gray.

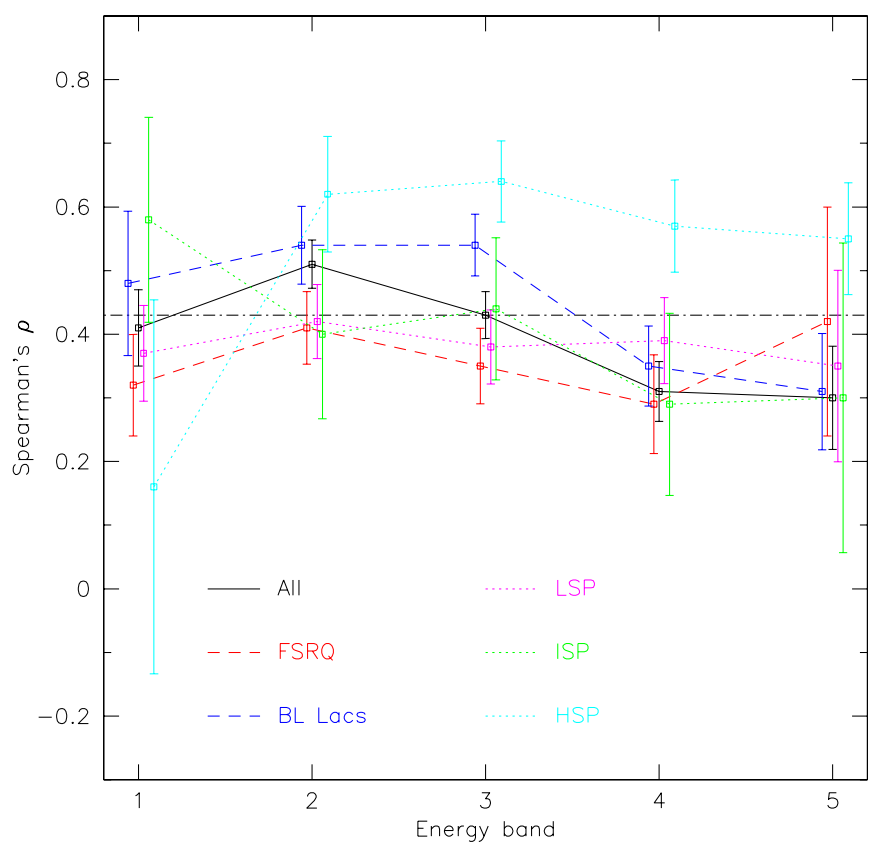

Figure 6. Correlation coefficient for the 1LAC sample as a function of the energy bands. Solid black line: all sources; dashed lines: sources divided by the optical type: red for FSRQs and blue for BL Lacs; dotted lines: sources divided by the spectral type (LSP in magenta, ISP in green, HSP in cyan). The dotdash black line shows as a reference the value of $\rho$ obtained using all sources and broadband gamma-ray flux. At each $x$-point (energy band), symbols are horizontally offset for improved clarity.

across the five energy bands and are connected with lines of different color and style for the various sub-populations: solid black line for the full 1LAC sample, dashed lines for optical type sub-groups (red for FSRQ and blue for BL Lacs), dotted lines for sub-groups defined by the spectral properties (magenta for LSP, green for ISP, cyan for HSP). The accuracy to which the correlation coefficients are determined, based on the number of sources and the strength of the correlation, is shown by the error bars, which correspond to the standard deviation for $\rho$, defined as $\sigma_{\rho}=\left(1-\rho^{2}\right) / \sqrt{N-1}$; although this standard deviation is formally defined only for the Pearson productmoment correlation coefficient $r$ (Wall \& Jenkins 2003), we extend it to our case, since the distribution of the Spearman $\rho$ for $N>30$ approaches that of the Pearson product-moment.

The Spearman correlation coefficient for all (599) sources is $\rho=0.43$. FSRQs and BL Lacs reveal different behaviors. In general, BL Lacs exhibit larger values of $\rho$ than FSRQs, both when the broadband gamma-ray energy flux is considered and in most of the single energy bands; for example, in the most populated energy band (for both populations, the $1-3 \mathrm{GeV}$ band, with 220 FSRQ and 214 BL Lacs), we find $\rho=0.54$ for BL Lacs and $\rho=0.35$ for FSRQs, although the difference is less significant in the other energy bands. Moreover, in FSRQs the correlation coefficient is quite stable across the various energy ranges (between $\rho=0.29$ and $\rho=0.42$ ), while BL Lacs display some evolution, with $\rho$ decreasing as fluxes at higher energy bands are considered. If one looks at the spectral type populations, HSPs are always the ones showing a tighter apparent correlation (except for the scarcely populated $100-300 \mathrm{MeV}$ band), and as high as $\rho=0.64$ in the $1-3 \mathrm{GeV}$ band.

\subsection{OVRO Sample}

The sources with OVRO data represent a 199 element subset of the 1LAC sample, going down to radio fluxes as low as 172 mJy (archival $8 \mathrm{GHz}$ value for $\mathrm{J} 1330+5202$, the source associated with 1FGL J1331.0+5202) and $64.7 \mathrm{mJy}(1$ year concurrent $15 \mathrm{GHz}$ value for J1725+1152). FSRQs outnumber BL Lacs by $120 / 69$. This sample provides the largest data set of concurrent radio measurements to the 1LAC fluxes and is therefore highly valuable in order to understand the implications of variability on the radio/gamma-ray correlation.

In particular, we are in the position of comparing the correlation coefficient not only among different source types and energy bands, but also to assess the differences that arise when we use concurrent data or not. In Table 3, we give the correlation coefficients: for the radio/gamma-ray flux densities using 
Table 3

Spearman Rank Correlation Coefficient $\rho$ for the Subset of the 1LAC Clean Sample Monitored by OVRO, Divided by the Source Type

\begin{tabular}{|c|c|c|c|c|c|c|c|c|c|c|c|c|}
\hline \multirow[t]{2}{*}{ Source Class } & \multicolumn{2}{|c|}{$E>0.1$} & \multicolumn{2}{|c|}{$0.1<E<0.3$} & \multicolumn{2}{|c|}{$0.3<E<1$} & \multicolumn{2}{|c|}{$1<E<3$} & \multicolumn{2}{|c|}{$3<E<10$} & \multicolumn{2}{|c|}{$10<E<100$} \\
\hline & $n$ & $\rho$ & $n$ & $\rho$ & $n$ & $\rho$ & $n$ & $\rho$ & $n$ & $\rho$ & $n$ & $\rho$ \\
\hline \multicolumn{13}{|c|}{$8 \mathrm{GHz}$ non simultaneous radio data } \\
\hline All sources & 199 & 0.27 & 97 & 0.40 & 163 & 0.41 & 179 & 0.25 & 134 & 0.15 & 40 & -0.04 \\
\hline FSRQ & 120 & 0.38 & 67 & 0.36 & 104 & 0.41 & 109 & 0.36 & 73 & 0.27 & 13 & 0.38 \\
\hline BL Lacs & 69 & 0.08 & 24 & 0.30 & 51 & 0.28 & 61 & 0.14 & 57 & 0.03 & 25 & -0.19 \\
\hline LSP & 131 & 0.30 & 77 & 0.42 & 113 & 0.41 & 120 & 0.29 & 89 & 0.28 & 24 & 0.16 \\
\hline ISP & 17 & 0.07 & 6 & 0.09 & 16 & 0.37 & 16 & 0.09 & 13 & -0.19 & 4 & 0.00 \\
\hline HSP & 10 & 0.73 & 6 & -0.02 & 10 & 0.64 & 10 & 0.79 & 10 & 0.67 & 9 & 0.67 \\
\hline \multicolumn{13}{|c|}{$15 \mathrm{GHz}$ concurrent radio data, 1 year mean values } \\
\hline All sources & 199 & 0.35 & 97 & 0.56 & 163 & 0.47 & 179 & 0.35 & 134 & 0.17 & 40 & -0.02 \\
\hline FSRQ & 120 & 0.48 & 67 & 0.56 & 104 & 0.54 & 109 & 0.49 & 73 & 0.35 & 13 & 0.57 \\
\hline BL Lacs & 69 & 0.13 & 24 & 0.41 & 51 & 0.22 & 61 & 0.14 & 57 & 0.00 & 25 & -0.23 \\
\hline LSP & 131 & 0.42 & 77 & 0.60 & 113 & 0.53 & 120 & 0.44 & 89 & 0.30 & 24 & 0.26 \\
\hline ISP & 17 & 0.29 & 6 & 0.66 & 16 & 0.54 & 16 & 0.29 & 13 & 0.20 & 4 & 0.40 \\
\hline HSP & 10 & 0.58 & 6 & -0.02 & 10 & 0.56 & 10 & 0.65 & 10 & 0.48 & 9 & 0.50 \\
\hline \multicolumn{13}{|c|}{$15 \mathrm{GHz}$ concurrent radio data, 1 year peak values } \\
\hline All sources & 199 & 0.28 & 97 & 0.56 & 163 & 0.41 & 179 & 0.27 & 134 & 0.11 & 40 & -0.06 \\
\hline FSRQ & 120 & 0.43 & 67 & 0.55 & 104 & 0.51 & 109 & 0.41 & 73 & 0.31 & 13 & 0.59 \\
\hline BL Lacs & 69 & -0.01 & 24 & 0.42 & 51 & 0.11 & 61 & 0.06 & 57 & -0.07 & 25 & -0.35 \\
\hline LSP & 131 & 0.42 & 77 & 0.60 & 113 & 0.52 & 120 & 0.43 & 89 & 0.30 & 24 & 0.30 \\
\hline ISP & 17 & 0.28 & 6 & 0.66 & 16 & 0.45 & 16 & 0.36 & 13 & 0.18 & 4 & 0.40 \\
\hline HSP & 10 & 0.13 & 6 & 0.09 & 10 & 0.16 & 10 & 0.16 & 10 & 0.12 & 9 & 0.08 \\
\hline \multicolumn{13}{|c|}{$15 \mathrm{GHz}$ non-concurrent radio data, 9 day mean values } \\
\hline All sources & 199 & 0.33 & 97 & 0.48 & 163 & 0.42 & 179 & 0.31 & 134 & 0.16 & 40 & -0.06 \\
\hline FSRQ & 120 & 0.45 & 67 & 0.49 & 104 & 0.51 & 109 & 0.47 & 73 & 0.35 & 13 & 0.34 \\
\hline BL Lacs & 69 & 0.13 & 24 & 0.24 & 51 & 0.15 & 61 & 0.11 & 57 & 0.01 & 25 & -0.20 \\
\hline LSP & 131 & 0.40 & 77 & 0.51 & 113 & 0.46 & 120 & 0.39 & 89 & 0.29 & 24 & 0.12 \\
\hline ISP & 17 & 0.12 & 6 & 0.43 & 16 & 0.31 & 16 & 0.06 & 13 & 0.10 & 4 & 0.80 \\
\hline HSP & 10 & 0.37 & 6 & 0.09 & 10 & 0.53 & 10 & 0.39 & 10 & 0.24 & 9 & 0.32 \\
\hline
\end{tabular}

Note. For each energy band ( $E$ measured in $\mathrm{GeV}$ ), the number of significant sources $n$ and the correlation coefficient $\rho$ are given.

historical radio flux densities at $8 \mathrm{GHz}$; the mean and peak flux density value at $15 \mathrm{GHz}$ calculated over the first 11 months of activity of the LAT; and an average $15 \mathrm{GHz}$ flux calculated over a one-week interval after the first 11 months of activity of the LAT (specifically, the period between 2010 January 23 and 31).

Figures 7-9 show the scatter plots of the concurrent radio and gamma-ray fluxes, using mean values for the radio flux density. As for the 1LAC case, we show three collections of scatter plots: radio versus gamma-ray energy flux for all sources, FSRQ, BL Lacs, LSP, ISP, and HSP sources in Figure 7, radio versus gamma-ray photon flux for all sources, FSRQ, and BL Lacs in Figure 8, and for LSP, ISP, and HSP sources in Figure 9. Finally, the trend of $\rho$ as a function of energy band for the various sub-classes is shown in Figure 10, with the same notation as in Figure 6.

Unlike for the larger 1LAC sample, in the sample with concurrently measured radio fluxes, FSRQs generally display larger values of $\rho$ than BL Lacs; as an example, in the 1-3 GeV energy band, $\rho_{\mathrm{FSRQ}}=0.48$ and $\rho_{\mathrm{BLL}}=0.13$. Moreover, the correlation coefficient for BL Lacs for the energy bands above $1 \mathrm{GeV}$ is consistent with no correlation, becoming even marginally negative in the $10-100 \mathrm{GeV}$ band. It has to be remembered that the OVRO sample is somewhat biased in favor of bright radio sources, so it contains relatively few BL Lacs, and in particular just a handful (10/199) of HSPs, as they are generally rather radio weak. Interestingly, the BL Lac curve falls below the three individual spectral type curves (LSP, ISP, HSP). We note that the sample also contains 41 sources (about $20 \%$ of the total) whose spectral type is unknown and that have almost uncorrelated radio and gamma-ray flux density. While this explains part of the difference between BL Lacs and $\mathrm{LSP}+\mathrm{ISP}+\mathrm{HSP}$, it is also important to warn that the $33 \mathrm{LSP} \mathrm{BL}$ Lacs do show systematically lower values of $\rho$ than the whole group of LSP sources (which is dominated by FSRQs).

As far as the radio variability is concerned, we find that for the whole sample the correlation coefficient with concurrent $15 \mathrm{GHz}$ data is always larger than that obtained using archival $8 \mathrm{GHz}$ data or non-concurrent $15 \mathrm{GHz}$ OVRO data. This result is mostly driven by the FSRQ population, while the less numerous BL Lac population does not seem to reveal significant differences between the use of concurrent or non-concurrent radio data. Finally, the use of the peak $15 \mathrm{GHz}$ flux density yields generally weaker correlations, in some cases even weaker than those found using non-concurrent data.

\subsection{Sources Significant in at least Four Energy Bands}

For both the 1LAC and the OVRO samples, we have considered in each energy range all the sources that were significant in that band. As a consequence of the different spectral characteristics of each individual source, the samples used to calculate the various coefficients have often little overlap between each 


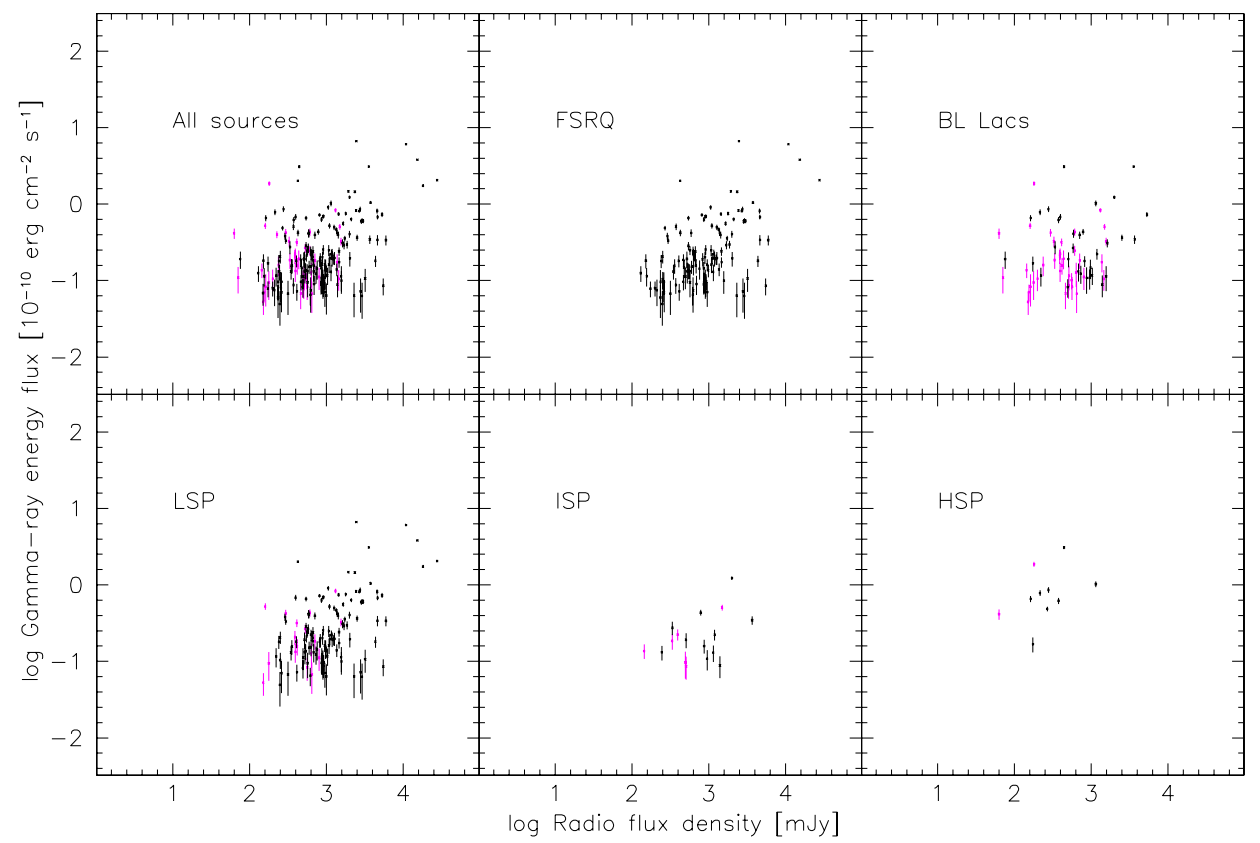

Figure 7. Broadband gamma-ray energy flux vs. concurrent $15 \mathrm{GHz}$ mean radio flux density for OVRO sources, divided by the source type. Top, from right to left: all AGNs, FSRQ, BL Lacs; bottom, from right to left: LSP, ISP, and HSP blazars. Sources with unknown redshift are shown in gray.

(A color version of this figure is available in the online journal.)

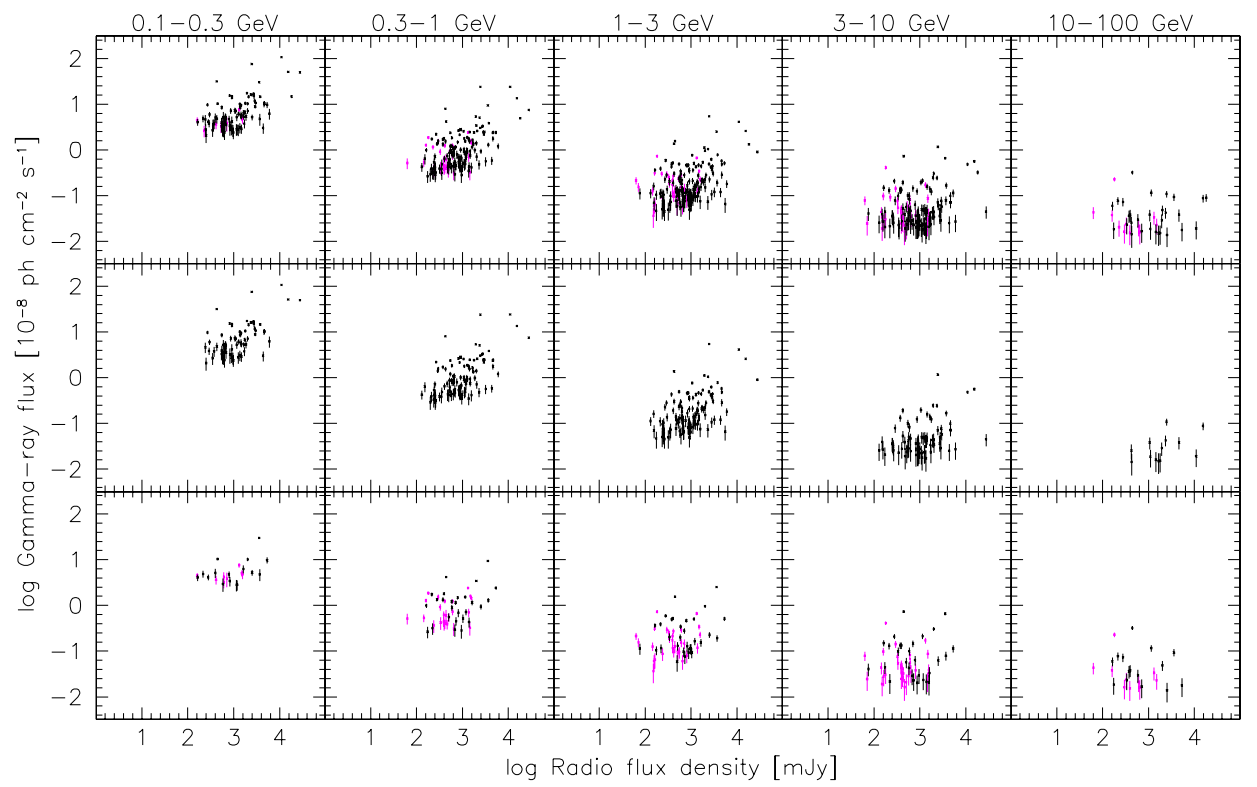

Figure 8. Gamma-ray photon flux vs. concurrent $15 \mathrm{GHz}$ mean radio flux density for OVRO sources, divided by the source optical type (top: all sources, middle: FSRQ, bottom: BL Lacs) and in energy bands. Sources with unknown redshift are shown in gray.

(A color version of this figure is available in the online journal.)

other (even within the same population), particularly in energy bands that are far apart.

For this reason, we have also considered a third case, the sample of sources that are significant in at least four of the five individual energy bands. In this way, we build a relatively bright, well defined, and sizable sample. This sample is composed of 192 sources, and both FSRQ (94 sources) and BL Lacs (84) are well represented.

As in the full 1LAC sample, BL Lacs have generally higher values of $\rho$ than FSRQ (e.g., $\rho_{\mathrm{BLL}}=0.54$ and $\rho_{\mathrm{FSRQ}}=0.29$ for the radio versus energy flux at $E>100 \mathrm{MeV}$ correlation). The individual values are reported in Table 4 and Figures 11-14, using the same notation as in the full 1LAC and OVRO cases.

If we look at the three groups defined by the synchrotron spectral properties, we find that the maximum of the correlation coefficient is obtained in the lowest energy band for LSP $(\rho=0.41$ between 100 and $300 \mathrm{MeV})$, in the $300 \mathrm{MeV}-1 \mathrm{GeV}$ for ISP $(\rho=0.63)$, and in the $1-3 \mathrm{GeV}$ for $\operatorname{HSP}(\rho=$ $0.74)$. Therefore-albeit with some overlap between the error bars-the higher the spectral frequency of the synchrotron spectral peak, the higher the energy at which the strongest apparent correlation is observed, and the higher the correlation coefficient itself. 


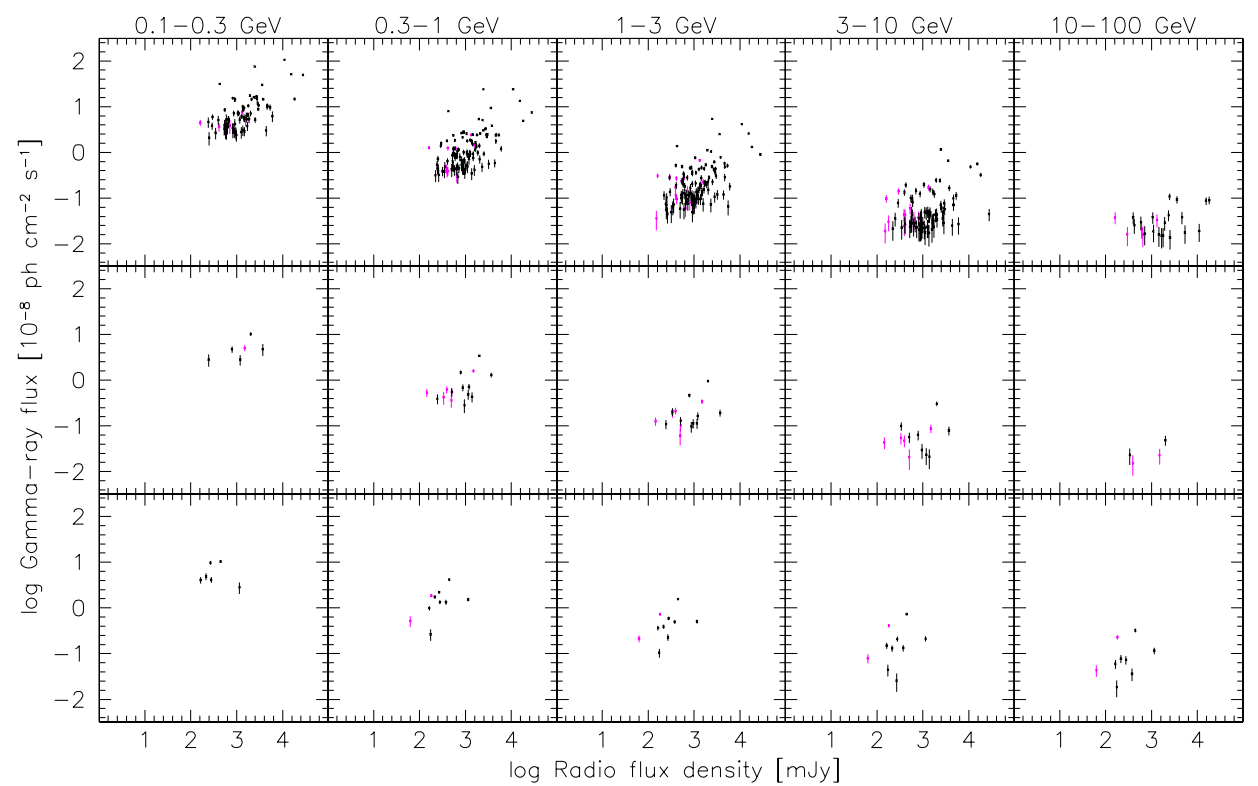

Figure 9. Gamma-ray photon flux vs. concurrent $15 \mathrm{GHz}$ mean radio flux density for OVRO sources, divided by the source spectral type (top: LSP, middle: ISP, bottom: HSP) and in energy bands. Sources with unknown redshift are shown in gray.

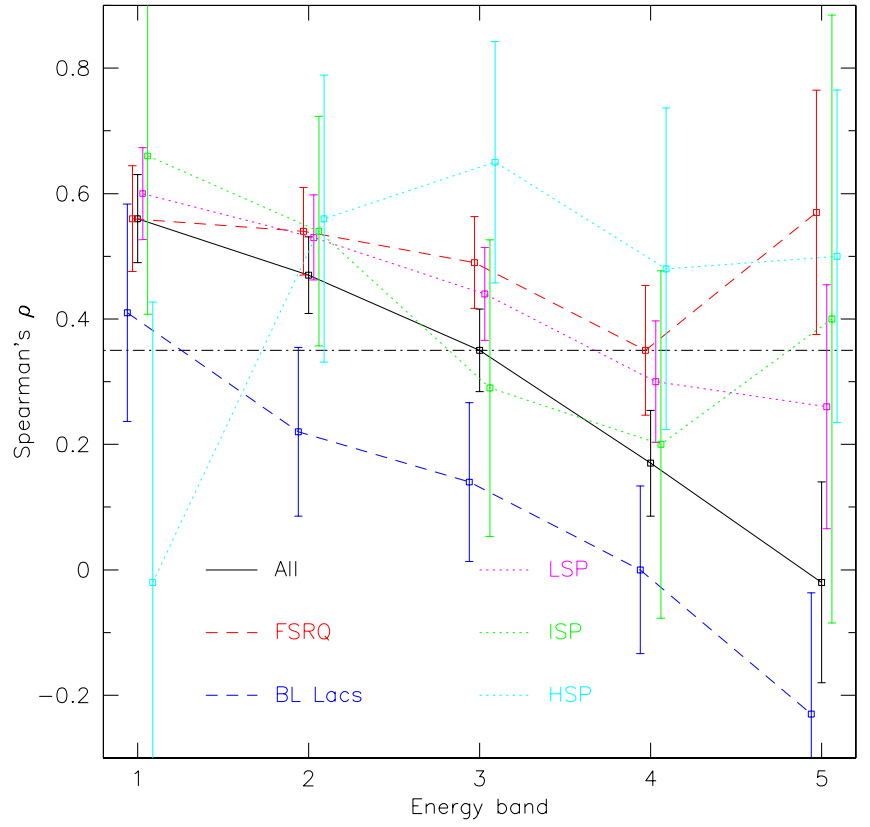

Figure 10. Correlation coefficients for the OVRO sample (concurrent radio and gamma-ray data) as a function of energy bands. Solid black line: all sources; dashed lines: sources divided by the optical type: red for FSRQs and blue for BL Lacs; dotted lines: sources divided by the spectral type (LSP in magenta, ISP in green, HSP in cyan). The dot-dash black line shows as a reference the value of $\rho$ obtained using all sources and broadband gamma-ray flux. At each $x$-point (energy band), symbols are horizontally offset for improved clarity.

\section{SIGNIFICANCE TESTS WITH THE METHOD OF SURROGATE DATA}

In order to quantitatively assess the significance of any apparent correlation between concurrent radio and gamma-ray flux densities of blazars in the presence of distance effects, we have used a test based on the method of surrogate data. In studying possible intrinsic correlations between flux densities in different bands the null hypothesis is that they are intrinsically uncorrelated (implicitly assuming that any apparent correlation is due to the observational errors and/or biases). In a frequentist approach, we investigate how frequently a sample of objects with intrinsically uncorrelated gamma/radio flux densities, similar to the sample at hand, will yield an apparent correlation as strong as the one seen in the data, when subjected to the same distance effects as our actual sample (see Pavlidou et al. 2011 for a more detailed description of the test).

In our test the strength of the apparent correlation is quantified by the Pearson product-moment correlation coefficient $r$, defined as

$$
r=\frac{\sum_{i=1}^{N}\left(X_{i}-\bar{X}\right)\left(Y_{i}-\bar{Y}\right)}{\sqrt{\sum_{i=1}^{N}\left(X_{i}-\bar{X}\right)^{2} \sum_{i=1}^{N}\left(Y_{i}-\bar{Y}\right)^{2}}} .
$$

Since it is not always straightforward to construct simulated samples with the exact same selection criteria as the data sample, we have used permutations of measured quantities. To simulate the effect of a common distance on intrinsically uncorrelated luminosities, we permute in luminosity space.

1. We split our sample in $N$ redshift bins, with $N$ determined so that each bin has at least $\sim 10$ sources. The separation in bins ensures that the luminosity and redshift distributions of the simulated samples approximate those in the real data, thus avoiding the introduction of biases not present in the data. Note however that, as we have shown in detail in Pavlidou et al. (2011), the significance of the correlation we find increases with increasing $N$ (the correlation becomes more significant), until it saturates for large enough $N$, provided that the number of sources is large enough.

2. In each redshift bin, from the measured radio and gammaray flux densities, we calculate radio and gamma-ray luminosities at a common rest-frame radio frequency and rest-frame gamma-ray energy. ${ }^{65}$

\footnotetext{
65 In order to implement the $K$-correction (project our calculated luminosities to a common rest-frame frequency in each band) we are using the historical radio spectral index $\alpha$ and the 1FGL photon index. The spectral index has been shown, at least at radio frequencies, to vary with flux (L. Fuhrmann et al. 2011, in preparation); however, as shown in Pavlidou et al. (2011), different choices in radio spectral indices do not have a large effect on the resulting correlation significance, as the sources of interest generally have flat radio spectra and the relevant $K$-correction is small.
} 


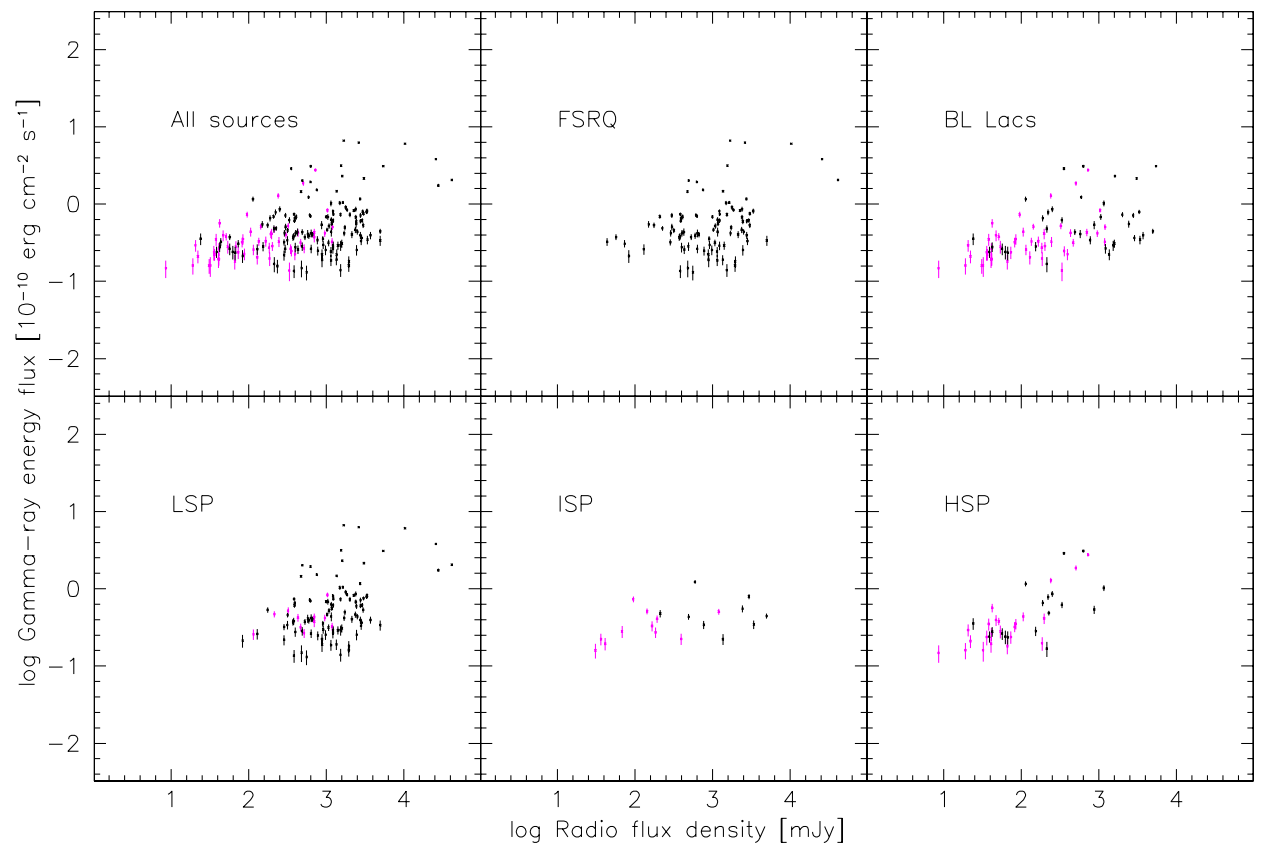

Figure 11. Broadband gamma-ray energy flux vs. $8 \mathrm{GHz}$ archival radio flux density for sources in the 1LAC with a detection in at least four energy bands, divided by the source type. Top, from right to left: all AGNs, FSRQ, BL Lacs; bottom, from right to left: LSP, ISP, and HSP blazars. Sources with unknown redshift are shown in gray. (A color version of this figure is available in the online journal.)

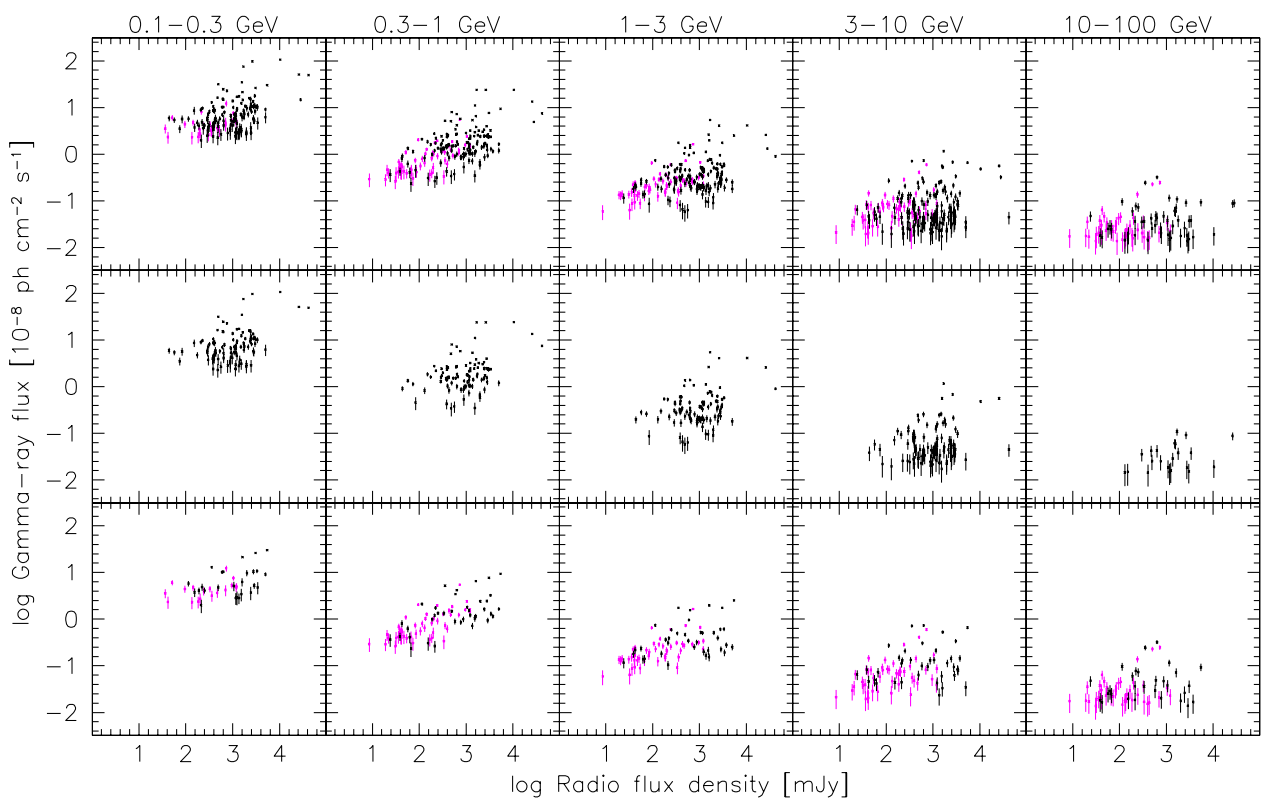

Figure 12. Gamma-ray photon flux vs. $8 \mathrm{GHz}$ radio flux density for sources in the 1LAC with a detection in at least four energy bands, divided by the source optical type (top: all sources, middle: FSRQ, bottom: BL Lacs) and in energy bands. Sources with unknown redshift are shown in gray.

(A color version of this figure is available in the online journal.)

Table 4

Spearman Rank Correlation Coefficient $\rho$ for 1LAC Sources with a Detection in at least Four Energy Bands, Divided by the Source Type

\begin{tabular}{|c|c|c|c|c|c|c|c|c|c|c|c|c|}
\hline \multirow[t]{2}{*}{ Source Class } & \multicolumn{2}{|c|}{$E>0.1$} & \multicolumn{2}{|c|}{$0.1<E<0.3$} & \multicolumn{2}{|c|}{$0.3<E<1$} & \multicolumn{2}{|c|}{$1<E<3$} & \multicolumn{2}{|c|}{$3<E<10$} & \multicolumn{2}{|c|}{$10<E<100$} \\
\hline & $n$ & $\rho$ & $n$ & $\rho$ & $n$ & $\rho$ & $n$ & $\rho$ & $n$ & $\rho$ & $n$ & $\rho$ \\
\hline All sources & 192 & 0.45 & 149 & 0.45 & 192 & 0.60 & 192 & 0.44 & 192 & 0.15 & 94 & 0.21 \\
\hline FSRQ & 94 & 0.30 & 93 & 0.34 & 94 & 0.30 & 94 & 0.24 & 94 & 0.15 & 22 & 0.42 \\
\hline BL Lacs & 84 & 0.55 & 44 & 0.48 & 84 & 0.72 & 84 & 0.62 & 84 & 0.41 & 67 & 0.23 \\
\hline LSP & 107 & 0.40 & 102 & 0.42 & 107 & 0.39 & 107 & 0.33 & 107 & 0.24 & 33 & 0.29 \\
\hline ISP & 20 & 0.46 & 15 & 0.52 & 20 & 0.63 & 20 & 0.26 & 20 & 0.16 & 12 & 0.02 \\
\hline HSP & 41 & 0.71 & 12 & 0.16 & 41 & 0.65 & 41 & 0.74 & 41 & 0.64 & 40 & 0.64 \\
\hline
\end{tabular}

Note. For each energy band ( $E$ measured in $\mathrm{GeV}$ ), the number of significant sources $n$ and the correlation coefficient $\rho$ are given. 


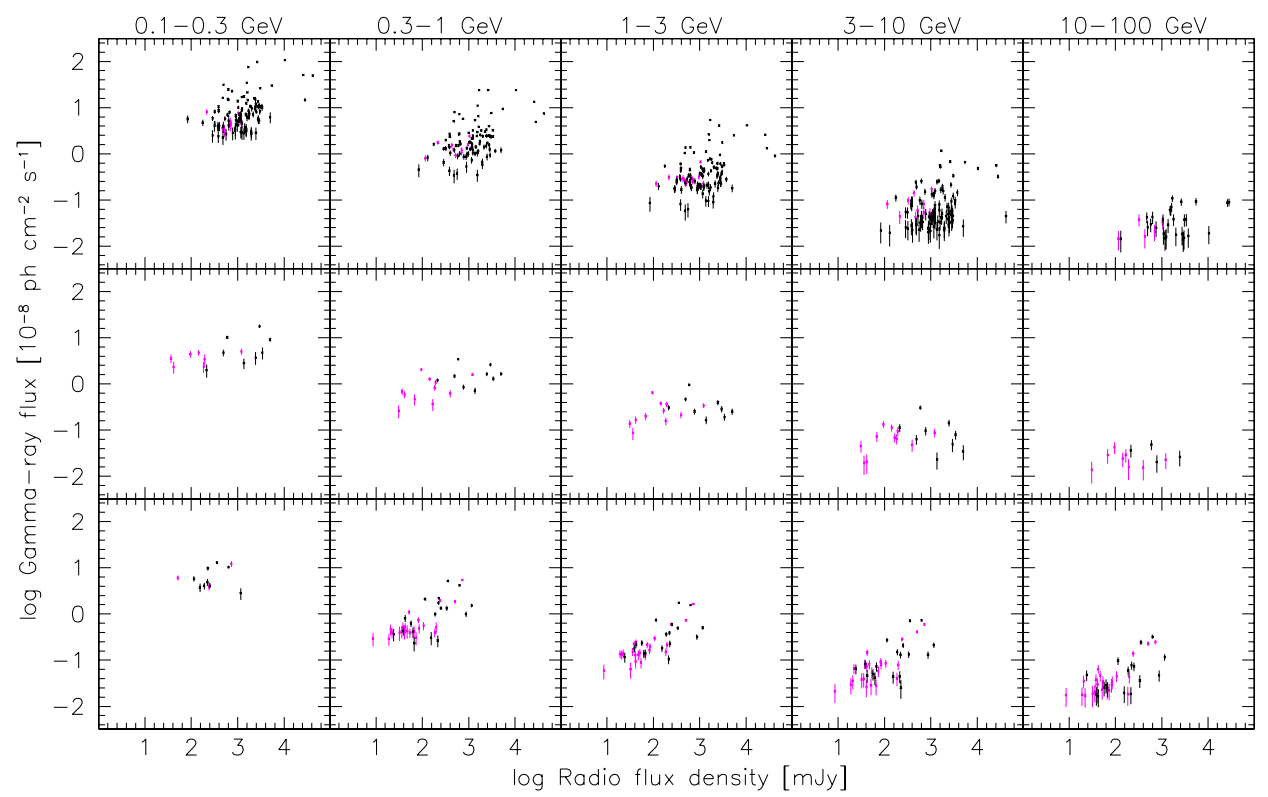

Figure 13. Gamma-ray photon flux vs. $8 \mathrm{GHz}$ radio flux density for sources in the $1 \mathrm{LAC}$ with a detection in at least four energy bands, divided by the source spectral type (top: LSP, middle: ISP, bottom: HSP) and in energy bands. Sources with unknown redshift are shown in gray.

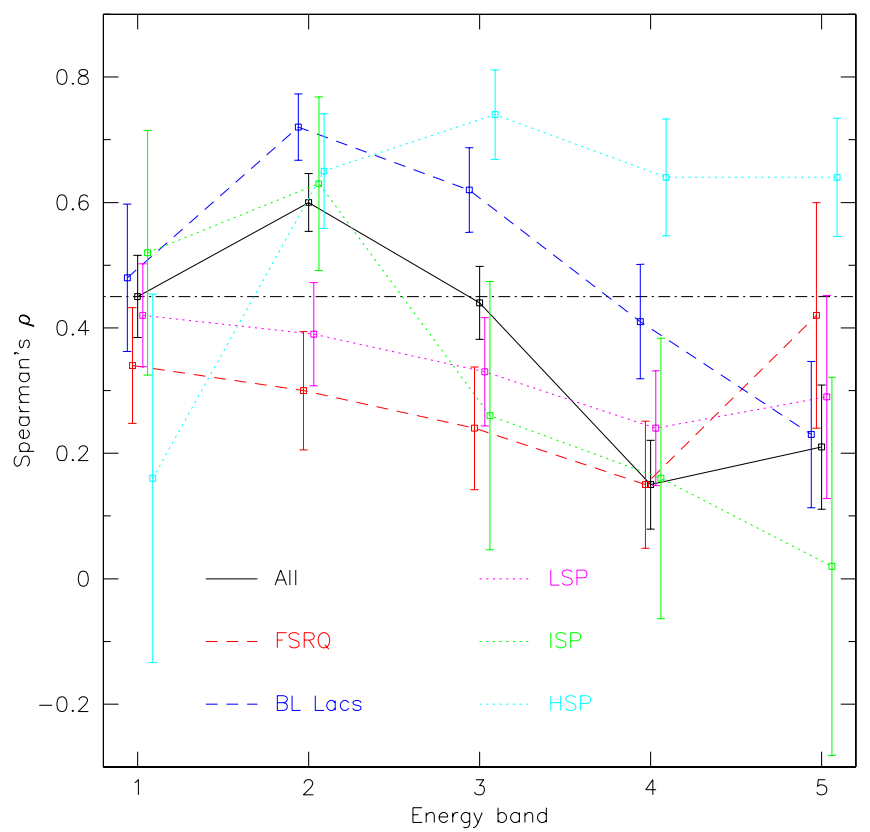

Figure 14. Correlation coefficient for sources in the 1LAC with a detection in at least four energy bands, as a function of the energy bands. Solid black line: all sources; dashed lines: sources divided by the optical type: red for FSRQs and blue for BL Lacs; dotted lines: sources divided by the spectral type (LSP in magenta, ISP in green, HSP in cyan). The dot-dash black line shows as a reference the value of $\rho$ obtained using all sources and broadband gamma-ray flux. At each $x$-point (energy band), symbols are horizontally offset for improved clarity.

3. We permute the evaluated luminosities to simulate objects with intrinsically uncorrelated radio/gamma luminosities.

4. We assign a common redshift (one of the redshifts of the objects in the bin, randomly selected) to each luminosity pair and return to flux-density space. Returning to fluxdensity space allows us to avoid Malmquist bias; assigning a common redshift allows us to simulate the commondistance effect on uncorrelated luminosities. In addition, by permuting in luminosity space we are guaranteed that the simulated samples have the same luminosity dynamical range as our actual sample.

5. To avoid apparent correlations induced by a single very bright or very faint object much brighter or fainter than the objects in our actual sample, we reject any fluxdensity pairs where one of the flux densities is outside the flux-density dynamical range in our original sample. The rejection rate is however very low for $N \geqslant 3$, and it decreases with increasing $N$.

Using a number of flux density pairs equal to the number of objects in our actual sample, we calculate a value for $r$. We repeat the process a large number of times, and calculate a distribution of $r$-values for intrinsically uncorrelated flux densities. The fraction of the area under this distribution for $|r| \geqslant r_{\text {data }}$, where $r_{\text {data }}$ is the $r$-value for the observed flux densities, is the probability to have obtained an apparent correlation at least as strong as a the one seen in the data from a sample with intrinsically uncorrelated gamma-ray/radio emission. This quantifies the statistical significance of the observed correlation.

Our results for all the correlations discussed in the present paper are shown in Tables 5 (full 1LAC sample), 6 (OVRO sample, using concurrent radio data), 7 (OVRO sample, using non-concurrent radio data), and 8 (sample of sources detected in at least four bands); for every case examined, we give the number of sources in the studied subset, the number of redshift bins used in the analysis, the Spearman correlation coefficient $\rho$, the value of the Pearson correlation coefficient $r$ of the data set, and the statistical significance of the apparent correlation, which we define as the fraction of simulated data sets with the same number of points, same common-distance, luminosity-range, and flux-range effects as the actual data set but no intrinsic correlation which had an absolute value of $r$ at least as big as the actual data set. The number of points in each data set studied generally differs from the number of points in the corresponding data set of Sections 3.1, 3.2, and 3.3, because for the surrogate data studies we only use sources for which the redshifts are known. In the scatter plots of Figures 3-5, 7-9, and 11-13, these sources are plotted with black points (while gray is used for sources with unknown $z$ ). For the same reason, the Spearman 
Table 5

Significances of Intrinsic Correlations After Common-distance Effects are Taken into Account, for Sources with Known Redshift, Using $8 \mathrm{GHz}$ Archival Data, as a Function of Source Type and $\gamma$-ray Energy Band

\begin{tabular}{|c|c|c|c|c|c|c|}
\hline Source Type & Energy Band & Number of Sources & Number of $z$-bins & Spearman $\rho$ & Pearson $r$ & Significance \\
\hline \multirow[t]{6}{*}{ All sources } & $E>0.1$ & 390 & 10 & 0.46 & 0.47 & $<10^{-7}$ \\
\hline & $0.1<E<0.3$ & 164 & 10 & 0.36 & 0.42 & $3.4 \times 10^{-5}$ \\
\hline & $0.3<E<1$ & 281 & 10 & 0.45 & 0.48 & $<10^{-7}$ \\
\hline & $1<E<3$ & 329 & 10 & 0.42 & 0.44 & $<10^{-7}$ \\
\hline & $3<E<10$ & 235 & 10 & 0.34 & 0.36 & $8.1 \times 10^{-6}$ \\
\hline & $10<E<100$ & 72 & 5 & 0.34 & 0.37 & 0.046 \\
\hline \multirow[t]{6}{*}{ FSRQ } & $E>0.1$ & 248 & 10 & 0.39 & 0.42 & $<10^{-7}$ \\
\hline & $0.1<E<0.3$ & 128 & 10 & 0.32 & 0.40 & $1.13 \times 10^{-4}$ \\
\hline & $0.3<E<1$ & 214 & 10 & 0.41 & 0.44 & $<10^{-7}$ \\
\hline & $1<E<3$ & 220 & 10 & 0.35 & 0.38 & $1.0 \times 10^{-7}$ \\
\hline & $3<E<10$ & 140 & 10 & 0.29 & 0.33 & $5.7 \times 10^{-4}$ \\
\hline & $10<E<100$ & 22 & 2 & 0.42 & 0.39 & 0.084 \\
\hline \multirow[t]{6}{*}{ BL Lacs } & $E>0.1$ & 116 & 10 & 0.63 & 0.62 & $<10^{-7}$ \\
\hline & $0.1<E<0.3$ & 27 & 3 & 0.42 & 0.43 & 0.034 \\
\hline & $0.3<E<1$ & 54 & 5 & 0.58 & 0.57 & $1.5 \times 10^{-4}$ \\
\hline & $1<E<3$ & 88 & 10 & 0.66 & 0.62 & $1.9 \times 10^{-6}$ \\
\hline & $3<E<10$ & 83 & 10 & 0.45 & 0.46 & $2.5 \times 10^{-3}$ \\
\hline & $10<E<100$ & 49 & 5 & 0.43 & 0.39 & 0.26 \\
\hline \multirow[t]{6}{*}{ LSP } & $E>0.1$ & 209 & 10 & 0.38 & 0.42 & $1.3 \times 10^{-6}$ \\
\hline & $0.1<E<0.3$ & 123 & 10 & 0.37 & 0.43 & $1.8 \times 10^{-4}$ \\
\hline & $0.3<E<1$ & 181 & 10 & 0.42 & 0.46 & $1.8 \times 10^{-7}$ \\
\hline & $1<E<3$ & 192 & 10 & 0.37 & 0.41 & $2.0 \times 10^{-6}$ \\
\hline & $3<E<10$ & 134 & 10 & 0.39 & 0.41 & $7.5 \times 10^{-4}$ \\
\hline & $10<E<100$ & 29 & 2 & 0.28 & 0.40 & 0.077 \\
\hline \multirow[t]{6}{*}{ ISP } & $E>0.1$ & 26 & 2 & 0.49 & 0.52 & 0.01 \\
\hline & $0.1<E<0.3$ & 9 & 1 & 0.62 & 0.55 & 0.13 \\
\hline & $0.3<E<1$ & 19 & 2 & 0.59 & 0.58 & 0.033 \\
\hline & $1<E<3$ & 22 & 2 & 0.45 & 0.44 & 0.082 \\
\hline & $3<E<10$ & 17 & 1 & 0.02 & 0.12 & 0.65 \\
\hline & $10<E<100$ & \multicolumn{5}{|c|}{ Not enough sources with redshift } \\
\hline \multirow[t]{6}{*}{ HSP } & $E>0.1$ & 73 & 7 & 0.49 & 0.68 & $3.0 \times 10^{-7}$ \\
\hline & $0.1<E<0.3$ & 9 & 1 & 0.15 & 0.003 & 0.99 \\
\hline & $0.3<E<1$ & 21 & 2 & 0.64 & 0.62 & 0.02 \\
\hline & $1<E<3$ & 48 & 4 & 0.66 & 0.74 & $7.0 \times 10^{-7}$ \\
\hline & $3<E<10$ & 45 & 5 & 0.56 & 0.70 & $2.6 \times 10^{-4}$ \\
\hline & $10<E<100$ & 35 & 3 & 0.59 & 0.71 & $5.5 \times 10^{-4}$ \\
\hline
\end{tabular}

correlation coefficient for the sets submitted to the surrogate data analysis is also different but consistent with the value shown in Tables 2-4.

In the case of large samples and relatively high correlation coefficients, the apparent correlations between radio and gamma-ray flux are found to be also intrinsically very significant: for example, the probability of the correlation between $E>100 \mathrm{MeV}$ gamma-ray flux and the $8 \mathrm{GHz}$ archival data arising due to common-distance effects or the limited flux and luminosity ranges examined is smaller than $10^{-7}$. However, for smaller subsets and weaker correlations (lower values of the correlation coefficients) the significance of the correlation cannot be established with such high confidence or not at all. A striking example is that of the correlation between $8 \mathrm{GHz}$ archival flux densities and 10-100 GeV fluxes for sources that were detected at least in four bands: the simulated data sets are more strongly correlated than the actual data set more than $30 \%$ of the time.

In Figure 15 we show, for three example cases, the distribution of the absolute value of the Pearson product-moment $r$ for simulated data sets. The value of $r$ for the actual data set in each case is indicated with an arrow. The three cases are selected so that they represent examples of low (top panel), medium (middle panel), and high (bottom panel) correlation significances. In some cases (as in the middle panel of these examples) the distribution of $r$ for simulated, intrinsically uncorrelated data sets peaks at a finite positive value even in flux space. This generally indicates a clustering of intrinsic luminosities around a specific value; these luminosities then are more frequently selected, even by chance, and when a common redshift is applied to them they result in a positive correlation coefficient, which then becomes more common than the zero value (for a more detailed discussion of this effect, see also Pavlidou et al. 2011).

The interpretation of the quoted significances requires some care, owing to the large number of subsamples (a total of 144) examined using the data shuffling technique. The quoted significances are a useful tool in comparing the significance of any apparent correlation between different subsamples, however the correlation significance in any single subsample is severely mitigated because of the issue of trials, e.g., any effect that occurs $5 \%$ of the time by chance would have a probability of 99.9\% to occur at least once in 144 independent trials; this probability becomes $76.3 \%$ for effects occurring by chance $1 \%$ of the time and $1 \%$ for effects occurring by chance only $10^{-4}$ of the time. Although the subsamples considered are, in fact, 
Table 6

Significances of Intrinsic Correlations After Common-distance Effects are Taken into Account, for Sources in the OVRO Sample with Known Redshift, Using $15 \mathrm{GHz}$ Concurrent Data, as a Function of Source Type and $\gamma$-ray Energy Band

\begin{tabular}{|c|c|c|c|c|c|c|}
\hline Source Type & Energy Band & Number of Sources & Number of $z$-bins & Spearman $\rho$ & Pearson $r$ & Significance \\
\hline All sources & $\begin{array}{c}E>0.1 \\
0.1<E<0.3 \\
0.3<E<1 \\
1<E<3 \\
3<E<10 \\
10<E<100\end{array}$ & $\begin{array}{r}160 \\
87 \\
136 \\
146 \\
104 \\
29\end{array}$ & $\begin{array}{r}10 \\
5 \\
10 \\
10 \\
10 \\
3\end{array}$ & $\begin{array}{l}0.39 \\
0.55 \\
0.50 \\
0.40 \\
0.22 \\
0.06\end{array}$ & $\begin{array}{l}0.46 \\
0.60 \\
0.57 \\
0.47 \\
0.28 \\
0.03\end{array}$ & $\begin{array}{c}9.0 \times 10^{-8} \\
1.3 \times 10^{-6} \\
3.0 \times 10^{-8} \\
2.7 \times 10^{-6} \\
0.021 \\
0.89\end{array}$ \\
\hline FSRQ & $\begin{array}{c}E>0.1 \\
0.1<E<0.3 \\
0.3<E<1 \\
1<E<3 \\
3<E<10 \\
10<E<100\end{array}$ & $\begin{array}{r}120 \\
67 \\
104 \\
109 \\
73 \\
13\end{array}$ & $\begin{array}{r}10 \\
6 \\
10 \\
10 \\
7 \\
1\end{array}$ & $\begin{array}{l}0.48 \\
0.56 \\
0.54 \\
0.49 \\
0.35 \\
0.57 \\
\end{array}$ & $\begin{array}{l}0.53 \\
0.61 \\
0.59 \\
0.54 \\
0.39 \\
0.46 \\
\end{array}$ & $\begin{array}{c}1.4 \times 10^{-7} \\
9.0 \times 10^{-8} \\
4.0 \times 10^{-8} \\
4.9 \times 10^{-6} \\
0.011 \\
0.11\end{array}$ \\
\hline BL Lacs & $\begin{array}{c}E>0.1 \\
0.1<E<0.3 \\
0.3<E<1 \\
1<E<3 \\
3<E<10 \\
10<E<100\end{array}$ & $\begin{array}{l}33 \\
16 \\
27 \\
31 \\
29 \\
15\end{array}$ & $\begin{array}{l}3 \\
1 \\
2 \\
3 \\
2 \\
1\end{array}$ & $\begin{array}{r}0.01 \\
0.36 \\
0.18 \\
0.07 \\
-0.08 \\
-0.15\end{array}$ & $\begin{array}{r}0.12 \\
0.44 \\
0.35 \\
0.22 \\
0.05 \\
-0.15 \\
\end{array}$ & $\begin{array}{l}0.57 \\
0.11 \\
0.17 \\
0.27 \\
0.90 \\
0.57\end{array}$ \\
\hline LSP & $\begin{array}{c}E>0.1 \\
0.1<E<0.3 \\
0.3<E<1 \\
1<E<3 \\
3<E<10 \\
10<E<100\end{array}$ & $\begin{array}{r}114 \\
70 \\
102 \\
106 \\
74 \\
19\end{array}$ & $\begin{array}{r}10 \\
7 \\
10 \\
10 \\
7 \\
1\end{array}$ & $\begin{array}{l}0.43 \\
0.59 \\
0.54 \\
0.47 \\
0.34 \\
0.30 \\
\end{array}$ & $\begin{array}{l}0.51 \\
0.62 \\
0.60 \\
0.54 \\
0.40 \\
0.38\end{array}$ & $\begin{array}{c}5.2 \times 10^{-6} \\
7.0 \times 10^{-7} \\
<10^{-7} \\
5.6 \times 10^{-6} \\
0.025 \\
0.11\end{array}$ \\
\hline ISP & $\begin{array}{c}E>0.1 \\
0.1<E<0.3 \\
0.3<E<1 \\
1<E<3 \\
3<E<10 \\
10<E<100\end{array}$ & $\begin{array}{r}11 \\
10 \\
10 \\
8\end{array}$ & $\begin{array}{l}1 \\
\text { Not enoug } \\
1 \\
1 \\
1 \\
\text { Not enoug }\end{array}$ & $\begin{array}{c}0.13 \\
\text { ces with redsh } \\
0.39 \\
0.31 \\
0.00 \\
\text { ces with redsh }\end{array}$ & $\begin{array}{l}0.51 \\
0.35 \\
0.11\end{array}$ & $\begin{array}{l}0.34 \\
0.20 \\
0.36 \\
0.80\end{array}$ \\
\hline HSP & $\begin{array}{c}E>0.1 \\
0.1<E<0.3 \\
0.3<E<1 \\
1<E<3 \\
3<E<10 \\
10<E<100\end{array}$ & $\begin{array}{l}8 \\
8 \\
8 \\
8\end{array}$ & $\begin{array}{l}1 \\
\text { Not enoug } \\
1 \\
1 \\
1 \\
\text { Not enoug }\end{array}$ & $\begin{array}{c}0.64 \\
\text { ces with redsh } \\
0.52 \\
0.76 \\
0.62 \\
\text { ces with redsh }\end{array}$ & $\begin{array}{l}0.44 \\
0.50 \\
0.44\end{array}$ & $\begin{array}{l}0.18 \\
\\
0.26 \\
0.20 \\
0.29\end{array}$ \\
\hline
\end{tabular}

not independent, the numbers above may serve as illustration that interpreting the here quoted significances at "face-value" can be misleading. However, for the largest samples considered here, we have found correlations with very high significance $\left(<10^{-7}\right)$, which remain very confident even in the face of the large number of trials.

We further remind that the method of surrogate data discussed above is applicable only to samples for which the redshifts of the sources are known. For this reason, when calculating significances for the apparent correlations of various subsamples, we discard sources for which the redshift is not known (most of such sources are BL Lacs). However, the omission of sources without known redshifts can affect the evaluated significance in two ways: by altering the redshift distribution of the sample and by reducing the number of sources. As a quantitative example of these effects, we have tested how our calculated significances change if we include the sources without known redshifts, and we assign redshifts to them in the following two ways: (1) assume that the missing redshifts have the same distribution as the known redshifts; in this case, we randomly select a redshift from sources of the same type (in most cases, BL Lacs) for the sources without redshifts; (2) assume that the missing redshifts have systematically higher values than the known redshifts; in particular, we assume that the distribution of the missing redshifts is that of known redshifts (for sources of the same type) translated to higher redshifts by $\Delta z=0.5$. We have tested these two cases for the sample of HSP blazars using $8 \mathrm{GHz}$ radio flux densities and gamma-ray flux in the $0.1-0.3 \mathrm{GeV}$ band.

The results for these test cases are shown in Figure 16. As an effect of the increased number of sources available for the test (47 instead of 21), the significance increases from $2.0 \times 10^{-2}$ to $4.2 \times 10^{-5}$ (if the sources without redshift follow the distribution generated with $\Delta z=0.5$ ) or even to $3.0 \times 10^{-8}$ (if they are distributed in the same way as the sources with a measured redshift). Note however that the results are substantially different depending on our choice of how to assign simulated redshifts, and for this reason we have not implemented this technique more extensively in our samples.

\section{DISCUSSION}

The sources in the 1LAC sample have an overall positive correlation between radio flux density and gamma-ray photon 
Table 7

Significances of Intrinsic Correlations After Common-distance Effects are Taken into Account, for Sources in the OVRO Sample with Known Redshift, Using $15 \mathrm{GHz}$ Non-concurrent Data, as a Function of Source Type and $\gamma$-ray Energy Band

\begin{tabular}{|c|c|c|c|c|c|c|}
\hline Source Type & Energy Band & Number of Sources & Number of $z$-bins & Spearman $\rho$ & Pearson $r$ & Significance \\
\hline All sources & $\begin{array}{c}E>0.1 \\
0.1<E<0.3 \\
0.3<E<1 \\
1<E<3 \\
3<E<10 \\
10<E<100\end{array}$ & $\begin{array}{r}160 \\
87 \\
136 \\
146 \\
104 \\
29\end{array}$ & $\begin{array}{r}10 \\
5 \\
10 \\
10 \\
10 \\
2\end{array}$ & $\begin{array}{r}0.36 \\
0.47 \\
0.45 \\
0.36 \\
0.21 \\
-0.06\end{array}$ & $\begin{array}{r}0.42 \\
0.51 \\
0.50 \\
0.42 \\
0.25 \\
-0.05\end{array}$ & $\begin{array}{c}1.9 \times 10^{-6} \\
7.2 \times 10^{-5} \\
2.0 \times 10^{-7} \\
4.1 \times 10^{-5} \\
0.045 \\
0.78\end{array}$ \\
\hline FSRQ & $\begin{array}{c}E>0.1 \\
0.1<E<0.3 \\
0.3<E<1 \\
1<E<3 \\
3<E<10 \\
10<E<100\end{array}$ & $\begin{array}{r}120 \\
67 \\
104 \\
109 \\
73 \\
13\end{array}$ & $\begin{array}{r}10 \\
6 \\
10 \\
10 \\
7 \\
1\end{array}$ & $\begin{array}{l}0.45 \\
0.49 \\
0.51 \\
0.47 \\
0.35 \\
0.34 \\
\end{array}$ & $\begin{array}{l}0.50 \\
0.54 \\
0.55 \\
0.51 \\
0.37 \\
0.30\end{array}$ & $\begin{array}{c}1.0 \times 10^{-6} \\
2.5 \times 10^{-6} \\
1.5 \times 10^{-6} \\
3.7 \times 10^{-5} \\
0.012 \\
0.31\end{array}$ \\
\hline BL Lacs & $\begin{array}{c}E>0.1 \\
0.1<E<0.3 \\
0.3<E<1 \\
1<E<3 \\
3<E<10 \\
10<E<100\end{array}$ & $\begin{array}{l}33 \\
16 \\
27 \\
31 \\
29 \\
15\end{array}$ & $\begin{array}{l}3 \\
1 \\
2 \\
3 \\
2 \\
1 \\
\end{array}$ & $\begin{array}{r}-0.02 \\
0.21 \\
0.05 \\
-0.01 \\
-0.07 \\
-0.19 \\
\end{array}$ & $\begin{array}{r}0.02 \\
0.19 \\
0.15 \\
0.05 \\
-0.01 \\
-0.22 \\
\end{array}$ & $\begin{array}{l}0.92 \\
0.49 \\
0.60 \\
0.80 \\
0.97 \\
0.41 \\
\end{array}$ \\
\hline LSP & $\begin{array}{c}E>0.1 \\
0.1<E<0.3 \\
0.3<E<1 \\
1<E<3 \\
3<E<10 \\
10<E<100\end{array}$ & $\begin{array}{r}114 \\
70 \\
102 \\
106 \\
74 \\
19\end{array}$ & $\begin{array}{r}10 \\
7 \\
10 \\
10 \\
7 \\
1\end{array}$ & $\begin{array}{l}0.40 \\
0.50 \\
0.46 \\
0.42 \\
0.33 \\
0.07\end{array}$ & $\begin{array}{l}0.46 \\
0.53 \\
0.51 \\
0.47 \\
0.34 \\
0.20\end{array}$ & $\begin{array}{c}7.1 \times 10^{-5} \\
6.4 \times 10^{-5} \\
2.8 \times 10^{-6} \\
6.7 \times 10^{-5} \\
0.07 \\
0.43\end{array}$ \\
\hline ISP & $\begin{array}{c}E>0.1 \\
0.1<E<0.3 \\
0.3<E<1 \\
1<E<3 \\
3<E<10 \\
10<E<100\end{array}$ & $\begin{array}{r}11 \\
10 \\
10 \\
8\end{array}$ & $\begin{array}{l}1 \\
\text { Not enoug } \\
1 \\
1 \\
1 \\
\text { Not enoug }\end{array}$ & $\begin{array}{c}0.14 \\
\text { ces with redsh } \\
0.36 \\
0.25 \\
0.00 \\
\text { ces with redsh }\end{array}$ & $\begin{array}{l}0.23 \\
\\
0.36 \\
0.17 \\
0.06\end{array}$ & $\begin{array}{l}0.50 \\
\\
0.38 \\
0.65 \\
0.90\end{array}$ \\
\hline HSP & $\begin{array}{c}E>0.1 \\
0.1<E<0.3 \\
0.3<E<1 \\
1<E<3 \\
3<E<10 \\
10<E<100\end{array}$ & $\begin{array}{l}8 \\
8 \\
8 \\
8\end{array}$ & $\begin{array}{l}1 \\
\text { Not enoug } \\
1 \\
1 \\
1 \\
\text { Not enoug }\end{array}$ & $\begin{array}{c}0.52 \\
\text { ces with redsh } \\
0.69 \\
0.57 \\
0.43 \\
\text { ces with redsh }\end{array}$ & $\begin{array}{l}0.48 \\
\\
0.50 \\
0.44 \\
0.31\end{array}$ & $\begin{array}{l}0.21 \\
\\
0.19 \\
0.27 \\
0.47\end{array}$ \\
\hline
\end{tabular}

flux, with a very high statistical significance as supported by the dedicated statistical analysis presented in Section 4. Moreover, the vast majority of the statistical tests run on the distribution of the gamma-ray and radio flux densities for the various source type/energy range combinations has also revealed some correlation (Section 3) with moderate-to-high statistical significance. Overall, this confirms the existence of a relationship between the emission in these two distant parts of the electromagnetic spectrum. This finding is consistent with other studies on the subject (Kovalev et al. 2009; Ghirlanda et al. 2010; Mahony et al. 2010). Most importantly, it has now been demonstrated to be robust against common-distance effects, and the effect of a limited flux and luminosity range.

In addition, the sensitivity of the LAT over three decades in energy range allows us to characterize a huge number of extragalactic gamma-ray sources across the gamma-ray band and to clarify some details of the relationship. The quality of the radio data provided by the archives as well as from concurrent monitoring are also crucial for a better understanding of the general picture.

For instance, BL Lacs are underrepresented in analyses performed starting from samples with moderate or high radio flux density limits, such as the AT20GHZ (Ghirlanda et al. 2010; Mahony et al. 2010) and the MOJAVE (Kovalev et al. 2009), whereas they actually constitute more than half of the 1LAC. Thanks to the archival interferometric data obtained for the full sample, we have studied the radio/gamma-ray connection within the two blazar sub-populations separately with a large number of sources. Indeed, even when considered independently, 1LAC BL Lacs display a correlation between their radio and gamma-ray flux densities that is highly significant; the chance probability is, e.g., $<10^{-7}$ when considering the full energy band and $1.9 \times 10^{-6}$ in the $1-3 \mathrm{GeV}$ energy band (see Table 5). As the surrogate data method can only be applied to sources with a known distance, it would be desirable to have more redshifts available for BL Lacs in order to improve the significance of this correlation also for other sub-bands. However, even with the lack of more redshift measurements, the conclusions implied from Figure 16 make one expect that such significance is no less than that of FSRQs or even higher given the larger value of both Spearman's $\rho$ and Pearson $r$ for BL Lacs.

The finding of a high apparent correlation strength for BL Lacs is not only present in the full 1LAC, but is also 
Table 8

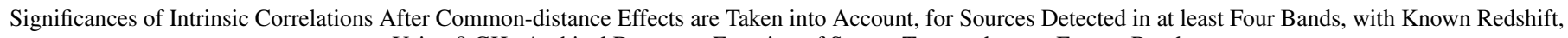
Using $8 \mathrm{GHz}$ Archival Data, as a Function of Source Type and $\gamma$-ray Energy Band

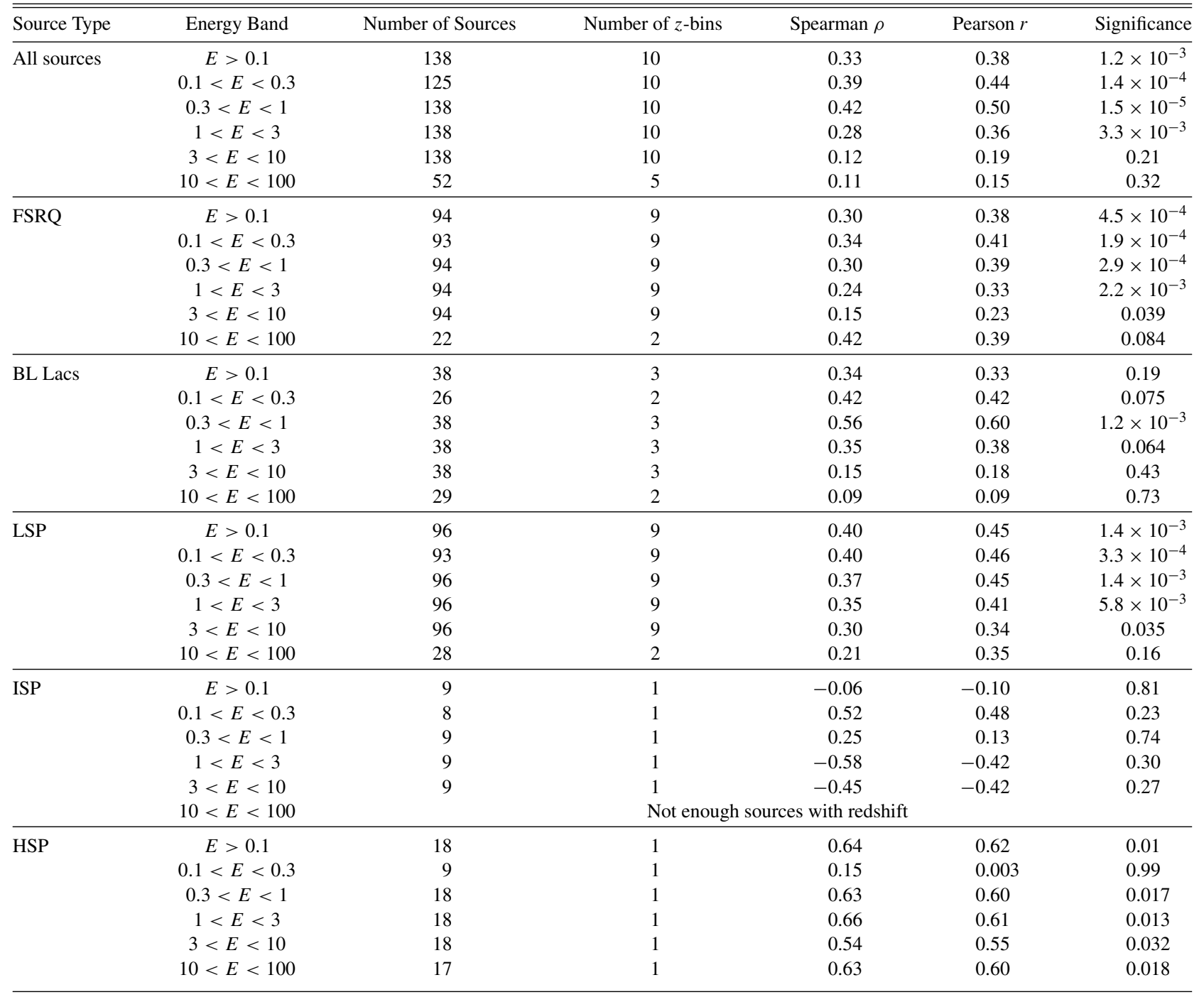

present-and actually with higher values of $\rho$-when one considers the results obtained for the sample of sources detected in at least four bands (Section 3.3). This sub-sample probably provides the most robust results, for two reasons. First, the $\rho$ values are obtained by considering largely overlapping samples in each energy bin; second, since these are moderately bright gamma-ray sources (they would not be significant in 4/5 energy bands otherwise), the fluxes are better constrained and we are not too close to the detection limit. Still, we caution that the four-band sample may not be a fully representative sample of the whole gamma-ray sky, as it is about $1 / 3$ of the 1LAC.

As far as the OVRO sample is concerned, it seems to have yielded somewhat different details of the overall picture with respect to the full 1LAC. This is not entirely surprising, as the two samples represent different populations: the OVRO sample is generally brighter compared to the whole 1LAC set, and FSRQs are more strongly represented. In any case, the availability of the large, long-term, high-cadence monitored OVRO sample is of great importance in the assessment of the role of variability on the radio-gamma connection. The OVRO data clearly reveal, for the first time, that concurrent radio fluxes are more strongly correlated with gamma-ray fluxes than archival data, even at the same frequency. For example, the significance of the correlation between radio and gamma-ray broadband fluxes for all sources increases from $1.9 \times 10^{-6}$ to $9.0 \times 10^{-8}$ when going from non-simultaneous to concurrent data. Increased significance is found also for most of the various combinations of source type and energy band. This was a long-expected result, which has finally been demonstrated. Interestingly, the peak radio flux density during the time of collection of gamma-ray data shows a weaker correlation than the one obtained using the mean values; it is actually even weaker than that of non-concurrent radio data. The fact that the strongest correlation is obtained for the time integral of the flux density in the two bands shows that the best correlation is between the overall energy dissipated in the two regimes.

A further advantage of such a large data set, which distinguishes our results from past work, is that it provides sufficient number of sources for good statistical analysis, even when we divide the sample in finer sub-groups, for example on the basis of the spectral properties in the synchrotron component of the spectral energy distribution (SED). The possibility of sub-grouping is interesting, particularly when we compare the results obtained dividing by optical type and position of the 


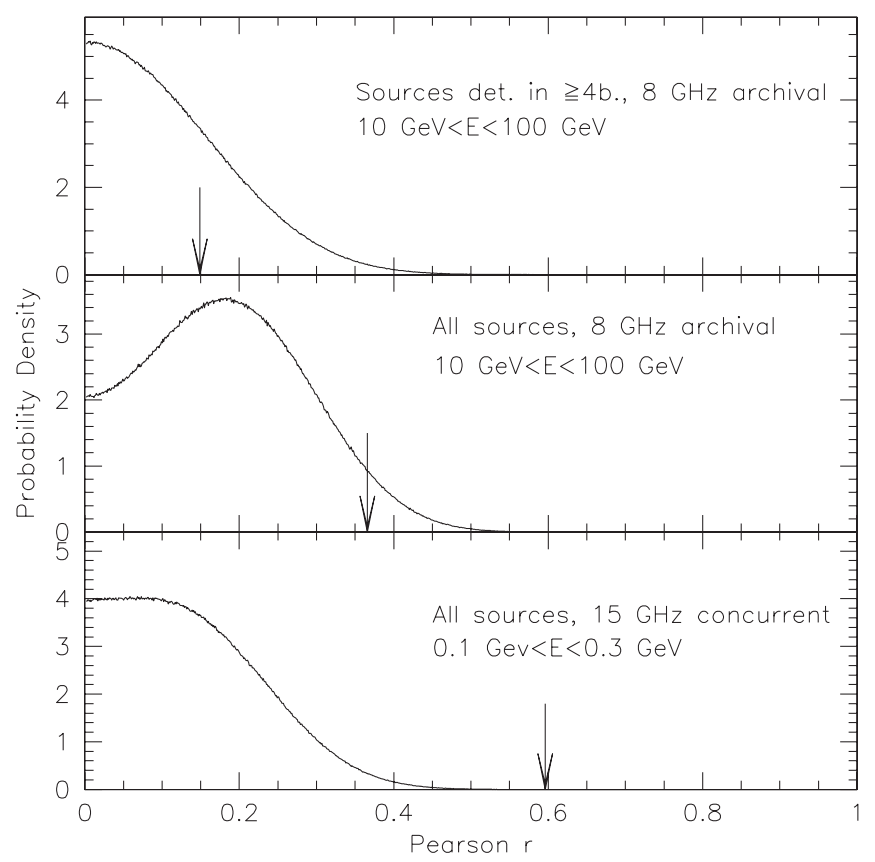

Figure 15. Probability density distributions of the absolute value of the Pearson product-moment $r$ for three simulated data sets, with low (top panel), medium (middle), and high (bottom) correlation significance.

peak of the low-energy component and/or considering each of the individual LAT energy bands. However, even if we are for the first time in the position of attempting such studies, we have to keep in mind that the statistical significance becomes inevitably lower when the samples are less populated, so the following discussion is certainly somewhat speculative.

First, the BL Lacs seem to follow a pattern of lower correlation coefficients when gamma rays of increasing energy are considered-with flux densities that become apparently uncorrelated (or even anti-correlated) in the highest energy band; this is particularly prominent in the OVRO sample. However, when the spectral types are considered separately, a pattern emerges with HSP always being the class with the strongest correlation in the 1-3, 3-10, and 10-100 GeV energy bands. ISP BL Lacs, on the other hand, show much weaker or absent correlation at high energy, which affects the total population of BL Lacs when considered as a whole. This effect becomes most prominent when the ISP/HSP population ratio is higher.

Second, LSP blazars are more difficult to characterize, since they are a mixed population of both BL Lacs and FSRQs. We note that FSRQ and LSP however do not always follow the same trend. It is thus likely that the radio and gamma-ray emission in FSRQ-LSP and the BL Lac-LSP are not produced by the same kind of process. This may or may not be related to the other well-known differences in the optical spectrum and in the accretion regime for these two populations.

The interpretation of the dependence of the correlation strength on the source type is therefore in general not straightforward. The fact that HSP sources show the strongest correlation could be related to the fact that these sources do not generally possess large amounts of extended emission, and even on parsec scales their jets are rather weak. So the interferometric flux density is probably more representative of the properties of the region where the gamma rays are produced. Moreover, in HSP sources the high energy component of the SED extends to the $\mathrm{TeV}$ band, so that the particles involved in the radio and $\mathrm{GeV}$

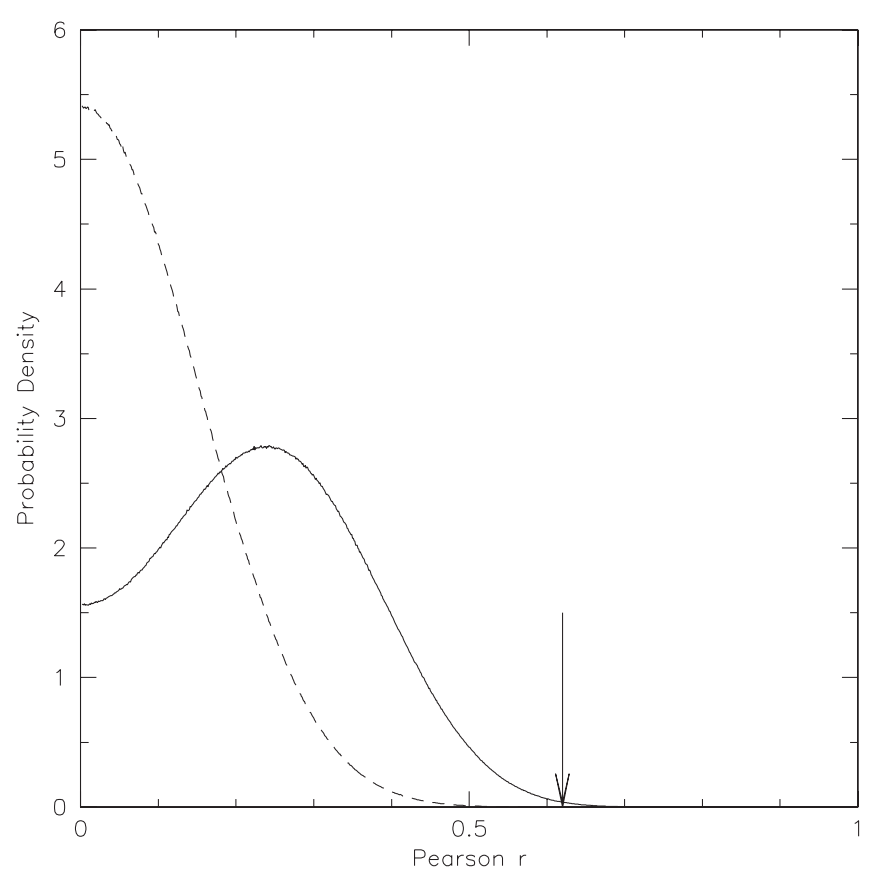

Figure 16. Probability density distribution of the absolute value of the Pearson product-moment $r$ for HSP blazars using $8 \mathrm{GHz}$ archival data and $0.1-0.3 \mathrm{GeV}$ gamma-ray flux density, assuming that the sources without redshift follow the same redshift distribution of the ones with known $z$ (dashed line) or with a mean shift of $\Delta z=0.5$ (solid line).

emission could be low energy electrons, unlike the LSP case where the $\mathrm{GeV}$ emission requires high energy particles.

The physical reason why even within a single population the correlation coefficient is quite strongly dependant on the considered energy band is even more difficult to interpret. For example, ISP blazars have a peak of correlation in the low energy band, while their radio and gamma-ray flux densities become essentially uncorrelated at the highest energies: sources of any radio flux density seem to produce more or less the same amount of gamma-ray photons. In other words, the radio-bright ISP blazars would have much softer gamma-ray spectra than the radio weak, which would be somewhat consistent with the picture in the blazar sequence Fossati et al. (1998); however, the same trend is not observed in other blazar classes.

Finally, one should be cautioned against correlations that are driven from a minority of very high (or very low) data points. For instance, Linford et al. (2011) find a flux-flux correlation for 50 gamma-ray FSRQs in the Very Long Baseline Array (VLBA) Imaging and Polarimetry Survey (VIPS; Helmboldt et al. 2007), but they also discover that the correlation disappears when the $10 \%$ brightest sources in the radio are discarded. In our 247 FSRQs sample, the effect is not quite as dramatic, with only a modest decrease of the correlation coefficient. As BL Lacs are entirely unaffected, however, this could still be an interesting clue about additional differences between the two classes.

\subsection{Luminosity Distributions}

Throughout this paper, we discuss the strength and the significance of radio/gamma correlations in terms of the flux density in each band. We do not discuss luminosity correlations, as the limited dynamical range in fluxes, combined with the aggregation of sources close to the flux limit and the squaredistance effect always induce a strong apparent correlation in luminosity space, whereas plots in the flux density plane give 
a better visual impression of the scatter involved. Moreover, it is possible to show analytically that the information in the flux/flux and luminosity/luminosity correlations is essentially degenerate.

However, from the physical point of view it is interesting to examine also the ranges and distributions of luminosities in the gamma-ray band and in the radio band, which are shown in Abdo et al. (2010c). The luminosity ranges probed by our sample extend over approximately two orders of magnitude in each band for BL Lacs and FSRQs, while they are more extended for other AGNs. Although there is significant overlap between the luminosities of different source types, FSRQs are generally more luminous than BL Lacs, which are in turn more luminous than the remaining AGNs (which includes radio galaxies and blazars of uncertain type); this result holds both for the radio and the gamma-ray bands. Therefore, we suggest that there is a lot of discovery space for sources of low gamma-ray luminosities, as the luminosity range of unclassified AGNs and radio galaxies extends more than two orders of magnitude fainter than BL Lacs and FSRQ in the gamma-ray band, but it is so far much less populated than the higher luminosity domain. It is possible that some of the fainter unassociated gamma-ray sources at high latitudes are AGNs in this luminosity range.

\section{CONCLUSIONS}

We have searched for a possible intrinsic correlation between gamma-ray and radio fluxes. We have found that such a correlation does exist, and it is statistically significant for the largest sample we have studied that includes all source types: the probability that it arises by chance (e.g., through commondistance effects, accentuated by the limited dynamical range of fluxes of the sample) is smaller than $10^{-7}$. The significance is also very high when FSRQ and BL Lacs are considered independently. However, the distribution of sources along the correlation has appreciable scatter (which can be typically an order of magnitude). Therefore, we strongly caution that any use of this intrinsic connection between radio and gamma-ray emission in statistical descriptions of the gamma-ray population, such as to obtain gamma-ray luminosity functions from radio luminosity functions, should be done with care and always accounting for the scatter involved. When comparing archival with concurrent data we find that the moderate significance of a correlation derived from the archival radio-gamma-ray sample increases appreciably when concurrent data are used.

The statistical significance of a correlation does not have a simple dependence on the apparent correlation strength. Various other factors play a role in an assessment of the significance of an apparent correlation, besides the tightness of the observed correlation itself. These include the following: (1) errors in the observed fluxes, (2) any biases, e.g., the presence of common-distance effects and flux limits, and (3) the number of sources in the sample. While underlying statistical errors (1) are inherent, in the present work we have explicitly accounted for biases (2). The sample size (3) affects the importance of "cosmic variance": a small sample with a significant correlation but appreciable statistical errors or scatter might happen to appear uncorrelated, and, conversely, any single incarnation of a small, uncorrelated sample might appear correlated by chance. Considering all of these factors, we have established high significance for some correlations but the same is not possible for very small subsamples.

We have studied the radio/gamma correlation for different subsets of blazars, and we have found that the apparent strength of the correlation depends on the type of blazar; in particular, BL Lacs have been shown to possess a high apparent correlation strength using the largest sample ever considered. The apparent strength of the correlation depends also on the epoch of observation, in that concurrent radio and gamma-ray measurements correlate better than data obtained at different epochs in the two bands.

Finally, the specific gamma-ray energy band over which the gamma-ray flux is calculated seems also to affect the strength of the correlation, with HSP blazars generally displaying a stronger correlation. The highest apparent correlation strength appears at higher gamma-ray energy for HSPs than for LSPs and ISPs. Both the specific energy bands and source types considered also impact the significance of the correlation. These results have been obtained thanks to the large number of AGNs detected by the Fermi-LAT, although in some of the considered correlations the number of sources is small. Therefore, a further increase in the number of objects in each sub-group, as that expected for the $2 \mathrm{LAC}$, is needed to improve the significance of individual results.

The Fermi LAT Collaboration acknowledges generous ongoing support from a number of agencies and institutes that have supported both the development and the operation of LAT as well as scientific data analysis. These include the National Aeronautics and Space Administration and the Department of Energy in the United States, the Commissariat à l'Energie Atomique and the Centre National de la Recherche Scientifique/ Institut National de Physique Nucléaire et de Physique des Particules in France, the Agenzia Spaziale Italiana and the Istituto Nazionale di Fisica Nucleare in Italy, the Ministry of Education, Culture, Sports, Science and Technology (MEXT), High Energy Accelerator Research Organization (KEK) and Japan Aerospace Exploration Agency (JAXA) in Japan, and the K. A. Wallenberg Foundation, the Swedish Research Council and the Swedish National Space Board in Sweden.

Additional support for science analysis during the operations phase is gratefully acknowledged from the Istituto Nazionale di Astrofisica in Italy and the Centre National d'Études Spatiales in France.

Facilities: Fermi, OVRO:40m, VLA, ATCA

\section{REFERENCES}

Abdo, A. A., Ackermann, M., Agudo, I., et al. 2010a, ApJ, 716, 30 Abdo, A. A., Ackermann, M., Ajello, M., et al. 2009, ApJ, 700, 597 (LBAS) Abdo, A. A., Ackermann, M., Ajello, M., et al. 2010b, ApJS, 188, 405 (1FGL) Abdo, A. A., Ackermann, M., Ajello, M., et al. 2010c, ApJ, 715, 429 (1LAC) Abdo, A. A., Ackermann, M., Ajello, M., et al. 2010d, ApJ, 722, 520 Abdo, A. A., Ackermann, M., Ajello, M., et al. 2010e, ApJ, 720, 912 Aharonian, F., Akhperjanian, A. G., Bazer-Bachi, A. R., et al. 2007, ApJ, 664, L71

Akujor, C. E., \& Garrington, S. T. 1995, A\&AS, 112, 235

Baars, J. W. M., Genzel, R., Pauliny-Toth, I. I. K., \& Witzel, A. 1977, A\&A, 61,99

Browne, I. W. A., Wilkinson, P. N., Jackson, N. J. F., et al. 2003, MNRAS, 341, 13

Condon, J. J., Cotton, W. D., Greisen, E. W., et al. 1998, AJ, 115, 1693

Danforth, C. W., Keeney, B. A., Stocke, J. T., Shull, J. M., \& Yao, Y. 2010, ApJ, 720,976

Dondi, L., \& Ghisellini, G. 1995, MNRAS, 273, 583

Elvis, M., Maccacaro, T., Wilson, A. S., et al. 1978, MNRAS, 183, 129

Feigelson, E. D., \& Berg, C. J. 1983, ApJ, 269, 400

Fossati, G., Maraschi, L., Celotti, A., Comastri, A., \& Ghisellini, G. 1998, MNRAS, 299, 433

Ghirlanda, G., Ghisellini, G., Tavecchio, F., \& Foschini, L. 2010, MNRAS, 407, 791 
Ghirlanda, G., Ghisellini, G., Tavecchio, F., Foschini, L., \& Bonnoli, G. 2011, MNRAS, 413, 852

Giroletti, M., Reimer, A., Fuhrmann, L., Pavlidou, V., \& Richards, J. L. 2010, arXiv: 1001.5123

Healey, S. E., Romani, R. W., Cotter, G., et al. 2008, ApJS, 175, 97

Healey, S. E., Romani, R. W., Taylor, G. B., et al. 2007, ApJS, 171, 61

Helmboldt, J. F., Taylor, G. B., Tremblay, S., et al. 2007, ApJ, 658, 203

Isobe, T. 1989, PhD thesis, Pennsylvania State Univ.

Komatsu, E., Dunkley, J., Nolta, M. R., et al. 2009, ApJS, 180, 330

Kovalev, Y. Y., Aller, H. D., Aller, M. F., et al. 2009, ApJ, 696, L17

Laurent-Muehleisen, S. A., Kollgaard, R. I., Ryan, P. J., et al. 1997, A\&AS, 122,235

Linford, J. D., Taylor, G. B., Romani, R. W., et al. 2011, ApJ, 726, 16

Mahony, E. K., Sadler, E. M., Murphy, T., et al. 2010, ApJ, 718, 587

Marchã, M. J. M., Browne, I. W. A., Impey, C. D., \& Smith, P. S. 1996, MNRAS, 281,425

Massaro, E., Giommi, P., Leto, C., et al. 2009, A\&A, 495, 691

Mauch, T., Murphy, T., Buttery, H. J., et al. 2003, MNRAS, 342, 1117
Mücke, A., Pohl, M., Reich, P., et al. 1997, A\&A, 320, 33

Mukherjee, R., Bertsch, D. L., Bloom, S. D., et al. 1997, ApJ, 490, 116

Myers, S. T., Jackson, N. J., Browne, I. W. A., et al. 2003, MNRAS, 341, 1

Nagar, N. M., Wilson, A. S., \& Falcke, H. 2001, ApJ, 559, L87

Padovani, P., Ghisellini, G., Fabian, A. C., \& Celotti, A. 1993, MNRAS, 260, L21

Pavlidou, V., et al. 2011, ApJ, submitted

Readhead, A. C. S., Lawrence, C. R., Myers, S. T., et al. 1989, ApJ, 346, 566

Richards, J. L., Max-Moerbeck, W., Pavlidou, V., et al. 2010, arXiv:1011.3111

Salamon, M. H., \& Stecker, F. W. 1994, ApJ, 430, L21

Schmitt, J. H. M. M. 1985, ApJ, 293, 178

Stecker, F. W., Salamon, M. H., \& Malkan, M. A. 1993, ApJ, 410, L71

Stocke, J. T., Morris, S. L., Gioia, I. M., et al. 1991, ApJS, 76, 813

Taylor, G. B., Healey, S. E., Helmboldt, J. F., et al. 2007, ApJ, 671, 1355

Tingay, S. J., Jauncey, D. L., King, E. A., et al. 2003, PASJ, 55, 351

Unger, S. W., Booler, R. V., \& Pedlar, A. 1984, MNRAS, 207, 679

Urry, C. M., \& Padovani, P. 1995, PASP, 107, 803

Wall, J. V., \& Jenkins, C. R. (ed.) 2003, Practical Statistics for Astronomers (Cambridge: Cambridge Univ. Press) 